\title{
28 Research Square \\ In vitro assembly, positioning and contraction of a division ring in minimal cells
}

\section{Shunshi Kohyama}

Max Plank Institution of Biochemistry https://orcid.org/0000-0002-7588-2488

\section{Adrian Merino-Salomon}

Max Planck Institute of Biochemistry

Petra Schwille ( $\nabla$ schwille@biochem.mpg.de)

Max Planck Institute of Biochemistry https://orcid.org/0000-0002-6106-4847

\section{Article}

Keywords:

Posted Date: February 17th, 2022

DOI: https://doi.org/10.21203/rs.3.rs-1284300/v1

License: (1) This work is licensed under a Creative Commons Attribution 4.0 International License.

Read Full License

Version of Record: A version of this preprint was published at Nature Communications on October 15th, 2022. See the published version at https://doi.org/10.1038/s41467-022-33679-x. 


\section{Abstract}

Constructing a minimal machinery for autonomous self-division of synthetic cells is a major goal of bottom-up synthetic biology. One paradigm has been the $E$. coli divisome, with the MinCDE protein system guiding assembly and positioning of a presumably contractile ring based on FtsZ and its membrane adaptor FtsA. Here, we demonstrate the full in vitro reconstitution of this machinery consisting of five proteins within lipid vesicles, allowing to observe the following sequence of events in real time: 1) Assembly of an isotropic filamentous FtsZ network, 2) its condensation into a ring-like structure, along with pole-to-pole mode selection of Min oscillations resulting in equatorial positioning, and 3 ) onset of ring constriction, deforming the vesicles from spherical shape. Besides demonstrating these essential features, we highlight the importance of decisive experimental factors, such as macromolecular crowding. Our results provide an exceptional showcase of the emergence of cell division in a minimal system, and may represent a major breakthrough towards developing a synthetic cell.

\section{Introduction}

Constructing a fully synthetic cell from defined biological molecules, such as DNA, proteins, and lipids, is one of the great aims of bottom-up synthetic biology ${ }^{1-5}$. Of the many essential features of cells, such as metabolism, replication, and interaction with the environment, cell division is probably the most tangible goal to be reached with a basic set of functional components, owing to the successful reconstitution and thorough mechanistic understanding of many division-related protein machineries ${ }^{6-8}$. One of the most advanced systems in this respect is the division machinery of the bacterium Escherichia coli ${ }^{9,10}$. In E. coli cells, three Min proteins called MinC, MinD, and MinE constitute a reaction-diffusion system that exhibits temporal oscillations between cell poles, so-called Min waves, generating a protein gradient that forms its maxima at the cell poles and minimum at mid-cell ${ }^{11,12}$. This protein gradient spatially regulates depolymerization of the division ring protein FtsZ, targeting proto-ring filaments to the middle of the cell by anchoring them to the membrane through FtsA and ZipA proteins, and constructing a primary division ring known as FtsZ-ring ${ }^{13,14}$ (Fig. 1a).

Remarkably, several of the self-organization processes of these protein systems have been reconstituted in vitro ${ }^{15,16}$. By analyzing the reconstituted Min gradient patterns and FtsZ polymerizationdepolymerization dynamics on supported lipid bilayers (SLBs) and inside lipid compartments, such as microdroplets and vesicles, in vitro studies have been extremely helpful in quantitatively understanding the molecular dynamics of these systems. Furthermore, lipid vesicles share essential features of cellular environments, such as transformable membrane boundaries and micron-scale confinement, and have also exhibited sufficient tolerance of physiological conditions including macromolecular crowding ${ }^{17,18}$ or high salt condition ${ }^{19}$. Therefore, reconstitution assays within lipid vesicles have been particularly attractive, although technically challenging ${ }^{20}$. For instance, due to the exceptionally low surface-tovolume ratio within microdroplets, spontaneous localization of MinE has to be modulated for a stable 
wave emergence ${ }^{21,22}$. In and on vesicles, FtsZ was able to deform membrane surfaces ${ }^{23-26}$ and dynamically reorganize dependent on membrane shape and anchoring features 26,27 .

Despite the importance of the interactions between the Min proteins and FtsZ for proper FtsZ-ring placement, the co-reconstitution of these proteins, especially in confined spaces, has rarely been accomplished so far ${ }^{28-31}$. This difficulty is mainly due to a large number of components and environmental factors to be controlled and their intrinsic complex dynamics that are still not fully understood $^{27,32}$.

Several studies attempted to reconstitute the Min waves and FtsZ structures via cell-free protein synthesis systems in vitro $24,25,31,33$. Although this approach avoids the complications imposed by protein purification, it is still challenging to express multiple functional proteins at the same time and in the right concentration ratios that have shown to be critical for the emergence of self-organization ${ }^{21,22,33,34}$. Also, the maximum protein yield is limited in cell-free expression systems ${ }^{35,36}$, while relatively high concentrations (up to $5 \mu \mathrm{M}$ ) of divisome proteins seem to be necessary to carry out full division in vivo $^{10,37,38}$. Indeed, none of the previous studies could express more than four division-related proteins within a single reactor to emerge Min oscillations together with FtsZ assembly, such that a visible spatial targeting of FtsZ filaments by Min waves, or condensation into a ring-like structure would be observed.

Herein, we successfully demonstrate Min wave-assisted FtsZ-ring assembly within lipid vesicles by two alternative approaches, in a fully controlled system with purified proteins, and by employing cell-free protein expression in a specifically tailored assay (Fig. 1a). We were able to follow in real time by timelapse imaging the origin, condensation, and equatorial placement of a minimal division ring-like FtsZ structure in giant unilamellar vesicles, more than ten times the size of bacterial cells. The reconstitution with purified proteins allowed a quantitative assessment of decisive factors and revealed that crowding environments are essential to forming FtsZ-ring structures. The reconstitution in a cell-free system, however, supported the time-sensitive sequential expression of five key divisome components: MinCDE, FtsA, and FtsZ, within lipid vesicles. Strikingly, this time-controlled series of events of FtsZ self-assembly and MinCDE self-organization resulted in a noticeable shape transformation of the spherical vesicle along with the Min-induced centric condensation of an originally isotropic FtsZ-FtsA meshwork. Our experiments reveal that such condensed FtsZ-ring structures are not only able to constrict the vesicle precisely in the middle, but also induce a clear symmetry breaking of Min oscillations upon deviation from spherical geometry, selecting a single pole-to-pole oscillation mode. This selected oscillation mode then intensifies further equatorial FtsZ condensation in a positive feedback mechanism. These exciting findings prove that complex spatiotemporal biological dynamics can indeed be reconstituted in vitro, marking a significant step towards constructing synthetic cells from the bottom-up.

\section{Results}




\section{Optimizing MinCDE reconstitution with respect to macromolecular crowding}

Recent experiments with reconstituted proteins revealed that macromolecular crowding significantly influences the functionality of divisome proteins ${ }^{21,39}$. In particular, the regulation of FtsZ by MinCDE, as well as the FtsZ condensation into pronounced ring structures is likely to depend critically on excluded volume effects ${ }^{28}$. Therefore, we first attempted an optimization of environmental conditions with respect to functionality of the main components, MinCDE and FtsZ. To this end, we adapted an experimental setup that stably generates Min waves via tuning MinE dynamics inside lipid compartments ${ }^{21,22}$. For standardized vesicle production, we used a double-emulsion transfer method ${ }^{33,40}$.

We first studied the protein concentration dependence of Min wave dynamics in vitro, which has been shown to be highly sensitive to MinD/MinE ratios on SLBs or in microdroplets $21,22,33,34$. Following established procedures, we successfully reconstituted MinCDE self-organization inside lipid vesicles (Fig. $1 \mathrm{~b}$ and $\mathrm{c}$ ). To identify the best conditions for co-reconstitution with FtsZ, we distinguished three different scenarios for Min protein localization: Dynamic waves at the membrane (further categorized into previously described modes in spherical vesicles: Pole-to-pole oscillations, traveling waves, and pulsing), stationary localization at the membrane, and stationary localization in the lumen ${ }^{33,41}$. In agreement with previous studies, we observed an overall higher prevalence of dynamic Min patterns, as compared to stationary concentrations in the lumen or at the membrane, when the MinDE ratios were roughly balanced (Fig. 1c). In contrast, spatiotemporal dynamics did not emerge when either MinD or MinE was in excess, resulting in stationary membrane localization at high MinD concentration or in the lumen at high MinE (Fig. 1c). We further analyzed the relative frequency of different modes among conditions with predominant Min dynamics ( $>65 \%$ ). Equimolar concentrations of MinDE at $3 \mu \mathrm{M}$ favored the generation of traveling waves and pole-to-pole oscillations (Supplementary Fig. 1). As pole-to-pole oscillations would be the desired mode for the spatiotemporal regulation of FtsZ at the membrane, we selected this MinDE concentration ratio as our standard condition for further assays.

Moreover, since previous studies indicated that macromolecular crowding environments enhance the polymerization of $\mathrm{FtsZ} \mathrm{Z}^{39,42-44}$ and intensify the regulation of FtsZ by Min waves into steeper gradients ${ }^{28}$, we figured that certain crowding conditions might improve functional co-reconstitution of both systems. The effects of macromolecular crowding on Min dynamics have been poorly addressed so $\operatorname{far}^{21,28}$. Therefore, we investigated the influence of different crowding conditions on Min wave dynamics inside lipid vesicles. To this end, we employed Dextran70 and Ficoll70, which are well-known and widely accepted macromolecular crowders in vitro $17,24,25,45,46$. We successfully reproduced the above-described MinCDE dynamics inside lipid vesicles under high crowding conditions (Fig. 1b and Supplementary Movie 1). Without crowders, Min proteins had yielded the three dynamic modes; pole-to-pole oscillation, traveling waves, and pulsing, at roughly $15 \%: 35 \%: 18 \%$ ratios at our standard MinCDE conditions (equimolar concentration of MinDE at $3 \mu \mathrm{M}$ and MinC at $0.5 \mu \mathrm{M}$ ). Intriguingly, after adding crowders, we observed a slight increase in the pole-to-pole oscillations, in spite of the unaltered spherical symmetry of the vesicle, 
which usually precludes mode selection ${ }^{41}$. Since stable pole-to-pole oscillations are required for correct placement of the FtsZ-ring in vivo ${ }^{11,12}$, any improvement in the prevalence of this mode would be highly beneficial for the assembly and placement of a functional division ring. However, the increase was not significant at particular crowding conditions, reaching up to 29\% of pole-to-pole oscillations at Ficoll70 $100 \mathrm{~g} / \mathrm{L}$, for instance (Fig. 1d). Therefore, we concluded that macromolecular crowders did not significantly affect the frequency of Min patterns under our experimental conditions. At the same time, we confirmed that a high percentage of the vesicles (70-80\%) showed Min dynamics under all crowding conditions, thus supporting the co-reconstitution of Min together with FtsZ.

\section{Co-reconstitution of MinCDE and FtsZ under optimized macromolecular crowding conditions}

Next, we investigated whether FtsZ can form bundles under crowding conditions inside lipid vesicles, as previously reported $23,27,44,47$. To demonstrate this, we employed FtsZ-Venus-mts, a widely used FtsZ mutant containing the YFP variant Venus before the membrane targeting sequence (mts) domain from MinD $16,23,48,49$. This mutant is able to bind the membrane independently of the interaction with the membrane anchor FtsA, which is challenging to reconstitute, due to their aggregative nature ${ }^{50}$. FtsZVenus-mts has greatly supported the in vitro reconstitution on membranes without dramatically affecting the FtsZ assembly dynamics ${ }^{48}$ (Fig. 2a) .

FtsZ was encapsulated inside lipid vesicles as described above. In the presence of crowders, a high proportion of FtsZ assembled into bundles, forming an isotropic mesh on the membrane. In contrast, the absence of crowders yielded only dot-like structures on the membrane (Fig. 2b, Supplementary Fig. 2a and $b$ ), similar to previous observations ${ }^{26}$. We quantitatively analyzed the effect of increasing concentrations of either Dextran70 or Ficoll70 on the formation of large-scale FtsZ structures (Supplementary Fig. 2a and c). The abundance of vesicles containing bundles and mesh structures increased considerably at $50 \mathrm{~g} / \mathrm{L}$ or higher crowding concentrations (Supplementary Fig. 2c), which led us to select that concentration range, in order to form FtsZ bundles efficiently.

Then, we co-reconstituted FtsZ and MinCDE systems within vesicles under crowding conditions (Fig. 2c). By analyzing the emergence of FtsZ structures inside lipid vesicles, we found that the formation of FtsZrings was highly related to the crowding concentration. The occurrence of FtsZ Mesh and FtsZ-rings increased with the concentration of crowders, and especially, it reached around $40 \%$ at $100 \mathrm{~g} / \mathrm{L}$ Dextran70, while we did not find any FtsZ-ring structure in the absence of crowders (Fig. 2e). Intriguingly, for $50 \mathrm{~g} / \mathrm{L}$ or higher concentrations of crowders, we observed an efficient condensation of previously isotropic FtsZ bundles into a single ring structure along with an equatorial positioning of this structure in the spherical vesicles, driven by pole-to-pole oscillations of the MinCDE wave (Fig. 2c). These FtsZ-rings were slightly fluctuating spatially around the middle region of the vesicle as a consequence of Min oscillations. However, the ring structures remained stably positioned in the middle of the vesicles for an extended period of about half an hour, which constitutes a significant accomplishment towards assembling a functional divisome (Fig. 2d and Supplementary Movie 2). 
In reverse, the presence of FtsZ in the equatorial region of the spherical vesicles yielded a significant increase in pole-to-pole Min oscillations under crowding conditions, indicating a tendency for mode selection by the presence of the ring-like FtsZ structures. The occurrence of Pole-to-pole oscillations under $100 \mathrm{~g} / \mathrm{L}$ Dextran70 increased up to $43 \%$, in agreement with the frequency of pronounced FtsZ-ring structures found under these conditions (Fig. 2f). Thus, we concluded that Min waves enhance and regulate FtsZ-ring assembly and placement with the help of macromolecular crowding in vitro. Moreover, there appears to be a positive feedback between Min oscillations and FtsZ positioning, resulting in the spontaneous breaking of spherical symmetry as a consequence of molecular self-organization within the vesicles.

\section{Increasing compositional complexity by PURE Cell-free expression}

Although the co-reconstitution of purified FtsZ-mts with MinCDE represents a major advance towards the assembly of a fully controlled minimal divisome, this system still lacks a potentially relevant degree of freedom in the regulation of FtsZ membrane attachment, which is in the cellular system conferred by anchoring through $\mathrm{FtsA}^{51,52}$. To investigate the potential relevance of this additional regulation feature, we set up the reconstitution of this more complex system inside lipid vesicles using the PURE cell-free expression system ${ }^{53,54}$. Since transcription and translation are the basis of cellular information processing, combining gene expression with a rudimentary division system is an intriguing alternative concept for the bottom-up construction of synthetic cells. Thus, several studies have previously been undertaken to incorporate the cell-free expression system into a minimal division system in vitro $24,25,31,33$. However, none of them has achieved reconstitution of the division ring placement system yet.

To conduct cell-free expression within lipid vesicles, we used our standard protocol of vesicle formation and observation. Additionally, we employed a Peltier stage mediated temperature control deviceto incubate the vesicle-containing chambers for cell-free protein synthesis. We first tested the effects of crowding on our experimental setup by expression of sfGFP. Compared to the expression without crowders, we confirmed a relatively lower expression yield of sfGFP under crowding environments, as previously reported $24,46,55,56$ (Fig. 3b and Supplementary Fig. 3). However, the sfGFP yield reached about $10 \mu \mathrm{M}$ with Ficoll70, whereas Dextran70 radically impaired expression. Because MinCDE, FtsZ and FtsA proteins would need up to $\sim 5 \mu \mathrm{M}$ of the expression yield, we employed the Ficoll70 as a crowding agent in cell-free expression experiments.

Then, we performed cell-free expression of FtsA with purified FtsZ-Alexa488 protein, which allowed us to integrate the wild-type FtsZ-FtsA cytoskeleton with the MinCDE system (Fig. 3a). In accordance with previous reports ${ }^{24,25}$, FtsZ formed bundles anchored through cell-free expressed FtsA on the membrane under the Ficoll70 crowding condition. Moreover, in addition to the FtsZ bundles at sufficient expression time (40-60 min), we also captured the process of the FtsZ bundle formation on the membrane along with the expression of FtsA over 40 min (Fig. 3c, Supplementary Fig. 4a, and Supplementary Movie 3). The time-lapse images show the dynamics of the FtsZ inside lipid vesicles that gradually formed mesh 
structures on the membrane, with the bundles branching into smaller ramifications (Fig. 3c). The localization of FtsZ molecules in the lumen of the vesicle gradually decreased by the expression of FtsA (Fig. 3c), while increase of FtsZ intensity was observed on the membrane (Supplementary Fig. 4b). Intriguingly, higher FtsZ concentrations on the membrane in larger vesicles were observed in larger vesicles, suggesting a correlation between size and membrane coverage (Supplementary Fig. 4c). Thus, we concluded that our experimental setup enables us to capture the dynamics of the reconstituted cytoskeleton system inside lipid vesicles via cell-free expression.

\section{Direct observation of FtsZ-ring formation inside vesicles}

To test whether the FtsZ-FtsA cytoskeleton system can also form FtsZ-ring structures inside vesicles that are spatially regulated by MinCDE waves, we combined the expression of FtsA and MinDE (Fig. 3a). Since the minDE operon structure from the $E$. coli genome successfully emerged Min patterns through PURE cell-free expression as previously reported ${ }^{33}$, we constructed the minDE operon DNA template as well. Cell-free expression from this operon DNA template verified the capacity of the MinDE expression system to emerge up to $80 \%$ of the Min patterns inside vesicles (Fig. 4c). We then attempted to express MinDE and FtsA with purified FtsZ-Alexa488 and mCherry-MinC to investigate the FtsZ dynamics under the regulation of Min waves.

As expected, the FtsZ structures dynamically transited in a time-dependent manner according to the emergence of Min wave patterns (Fig. 3d and Supplementary Movie 4). At the beginning of protein expression, neither FtsZ nor Min proteins formed any patterns on the membrane. Then, FtsZ gradually developed mesh structures on the membrane, similar to the sole expression of FtsA with FtsZ-alexa488. Strikingly, after FtsZ mesh formation, Min waves emerged predominantly as a pole-to-pole oscillations and instantly regulated FtsZ localization, leading FtsZ to form a ring-like structure at the middle of the vesicle. Thus, we confirmed that partially cell-free expressed FtsZ-FtsA and MinCDE system coordinate to assemble and correctly position FtsZ-ring structures inside lipid vesicles. However, the ring structure obtained like this was not stable enough, because the Min oscillations eventually deteriorated into traveling waves, and FtsZ followed the wave propagation pattern into antagonistic, but temporally unstable localization.

These time-dependent pattern changes of Min waves leading to a loss in mode-locked pole-to-pole oscillations were similar to the ones previously reported ${ }^{21}$. Herein, Min waves inside microdroplets showed pole-to-pole oscillation in the relatively early phase after Min wave emergence, but then deteriorated towards traveling waves over time. Indeed, some vesicles showed pole-to-pole oscillation only less than $5 \mathrm{~min}$ and then transited to traveling waves (Supplementary Fig. 4d). FtsZ followed this trend and formed pronounced ring structures only while pole-to-pole oscillation were present (Supplementary Fig. 4d). Thus, it became obvious that Min waves strictly govern FtsZ patterns, and more importantly, out of the two major dynamic Min patterns, only pole-to-pole oscillations, but not traveling waves, support stable FtsZ-ring formation.

\section{Regulation of MinCDE waves by fully expressed proteins}


After reconstitution of FtsZ-ring structures by partially cell-free expressed proteins, we expanded the system further to realize a reconstituted division system from entirely genetically encoded proteins. One of the challenges for this approach is that either one or two proteins must be fused with the Fluorescent Proteins (referred as FPs) to observe their dynamics by fluorescence microscopy. In this respect, E. coli FtsZ is known to lose its functionality by fusing tag proteins to the C-terminus ${ }^{57,58}$. Furthermore, an increase in the molecular weight of the FtsZ protein by conjugating average-size FPs decreases protein yield. On the other hand, since MinDE waves are highly sensitive to their concentration ratio, it needs to be validated that cell-free expressed MinCDE proteins can maintain ideal concentrations to emerge wave dynamics. Thus, we first reconstituted the partial systems (MinCDE vs. FtsZ-FtsA) to check the selforganization ability of cell-free expressed proteins (Fig. 4a).

Since the minDE operon DNA template was already optimized for cell-free expression, we decided to keep the $\min D E$ operon structure for the MinDE expression. Therefore, we additionally introduced mCherryMinC as the reporter of the Min wave localization and at the same time regulator of the FtsZ localization. As expected, co-expressed MinCDE proteins sustain Min wave emergence inside vesicles (Fig. $4 \mathrm{~b}$ and Supplementary Movie 5), implying that MinDE concentrations were still within a well-balanced range of co-expression. The kymographs revealed that Min wave patterns gradually appeared along with the synthesis of mCherry-MinC (Fig. 4b). As for MinDE co-expression, wave patterns dynamically transited from pole-to-pole oscillation to traveling waves. Moreover, extended time-lapse observation up to $2.5 \mathrm{~h}$ captured that Min waves eventually showed static patterns, which agreed with the emergence of static patterns after 2-4 h of MinDE expression ${ }^{33}$.

Compared to MinDE expression, full MinCDE co-expression indicated even lower efficiency and slower pattern emergence (Fig. 4c). This impaired efficiency of wave emergence might be due to the conflict of the protein synthesis resources between mCherry-MinC and MinDE templates. However, we confirmed that at least $60 \%$ of the vesicles contained Min patterns after $1 \mathrm{~h}$ co-expression of MinCDE proteins.

\section{Functional FtsZ-FP for FtsZ-FtsA reconstitution via cell-free expression}

Next, we tackled the re-assembly of FtsZ-FtsA from cell-free synthesized FtsA and FtsZ, which promised to be more complicated than the MinCDE system. In particular, the C-terminal conjugation with FPs impairs the functionality of FtsZ in vivo ${ }^{57,58}$, because interactions with division proteins occur mainly through the $\mathrm{C}$-terminal domain of $\mathrm{FtsZ} \mathrm{Z}^{59,60}$. To overcome this difficulty, we adapted a novel FtsZ mutant according to a previous report ${ }^{61}$, in which FPs are inserted into the G55-G56 position of FtsZ instead of Cterminal conjugation. These chimeric FtsZ-G55-FP-G56 mutants were reported as fully functional in vivo, and naturally, expected to have similar functionalities as wild-type FtsZ in vitro.

At the same time, the considerable gain in molecular weight from the fusion of FPs with wild-type FtsZ (FtsZ-FPs, $\sim 67 \mathrm{kDa}$ ) renders it difficult to implement a close-to-physiological concentration of FtsZ (1-6 $\mu \mathrm{M})^{10,37,38}$ using cell-free expression with respect to FtsA $(45 \mathrm{kDa}, 0.07-0.3 \mu \mathrm{M})^{37,38}$. Therefore, we varied 
the FtsZ and FtsA template concentrations to maintain the ideal protein proportions to form FtsZ bundles. After optimizing the template ratio ( $3 \mathrm{nM}$ for FtsZ and $1 \mathrm{nM}$ for FtsA), co-expressed FtsZ-G55Venus-Q56 and FtsA generated FtsZ mesh structures on the membrane (Fig. 4d). Simultaneously, we captured the mesh formation of FtsZ-G55-Venus-Q56 that showed to be similar to the FtsA-only expression with purified FtsZ-Alexa488 (Fig. 4e, Supplementary and Supplementary Movie 6), also demonstrating similar correlation of FtsZ intensity on the membrane and vesicle size (Supplementary Fig. $5 a$ and $b$ ). On the other hand, C-terminally fused FtsZ-Venus did not show preferential membrane localization, verifying that this chimeric FtsZ protein lacks this functionality (Fig. 4e and Supplementary Fig. 5c). Moreover, we checked the activity of purified FtsZ-G55-Venus-Q56 by SLBs and GTPase assay, confirming that FtsZ-G55-Venus-Q56 is active under our experimental conditions (Fig. 4f, Supplementary Fig. $5 d$, and e). Together with the reconstitution of the MinCDE system, we concluded that sub-division systems were faithfully reconstituted from cell-free expressed proteins inside lipid vesicles.

\section{Mid-cell placement and radial constriction of a minimal division ring}

Finally, we incorporated the co-expression of MinCDE, FtsZ and FtsA proteins within the same vesicles (Fig. 5a). After considering the concentration ratios of four templates to obtain ideal protein yields, we confirmed that upon co-expression of all five proteins, FtsZ-ring structures spatially self-assembled under the regulation of Min waves (Fig. $5 \mathrm{~b}$ and Supplementary Movie 7). As observed for the partial coexpression described above (Fig. 3d), FtsZ first assembled into isotropic mesh structures, which upon the onset of MinCDE oscillations spatially condensed into an FtsZ-ring structure in the middle of the vesicle (Fig. 5c). In turn, as observed for the purified proteins, the formation of the ring structure led to a breaking of spherical symmetry, which arrested the Min oscillations into a pronounced pole-to-pole mode.

Strikingly, we found that the FtsZ-ring assembly together with the pole-to-pole oscillations resulted in a marked deformation of the lipid vesicles away from spherical to a more rod-like shape, reflecting on a radial constriction of up to $19 \%$ (Fig. 5c, Supplementary Fig. 7b and Supplementary Movie 8). This kind of vesicle deformation by a central FtsZ-ring structure could also be observed for static bipolar localization of Min proteins (Supplementary Fig. 6a), implying that the static pattern of Min waves also supports FtsZ-ring assembly and placement. Time-lapse images suggest that in these cases, Min waves first appeared as pole-to-pole oscillations to form the FtsZ-ring, and later transformed into a static bipolar localization depending on MinDE concentrations. However, although we suspect that some sort of dissipative mechanism is at place in these vesicles, the spatial regulation of the FtsZ-ring structure to the middle of the vesicles seems to be preserved (Supplementary Fig. 6b). The surface plot of FtsZ intensities revealed that FtsZ was randomly distributed on the membrane but then formed and reinforced two peaks over time, corresponding to a ring-like structure and implying that the Min waves successfully reshaped FtsZ structures and stabilized them (Supplementary Fig. 6d). In addition, also non-deformed vesicles showed FtsZ mesh structures antagonistically localized against Min traveling waves (Supplementary Fig. $6 \mathrm{c}$ ). The fact that all three wave modes could be observed after MinCDE expression might reflect on differences of MinDE concentrations among vesicles (Fig. 4b). 
Evaluation of the size dependence of the FtsZ organization and deformation of vesicles revealed more features of the reconstituted minimal division system. The population of deformed vesicles with pronounced FtsZ-ring structure radically increased with the vesicle size, while relatively small vesicles $(\sim 12 \mu \mathrm{m})$ remained spherical (Fig. 5d). We found that the aspect ratios of the deformed vesicles and their original diameters were moderately correlated ( $r$-value $=-0.40$, Fig. 5 e). In several cases such as Fig. $5 c$, the aspect ratios diameter/length of the deformed vesicle approached about 0.75 after $3 \mathrm{~h}$ expression. Since we performed all experiments under isosmotic conditions, this suggests that the FtsZ-ring upon spatial condensation generated considerable forces to constrict the vesicles radially by up to 19\% (Supplementary Fig. 7). Based on this striking observation, it will be the key challenge for further investigations to develop a biophysical assay for directly measuring these forces, and thereby explore the force generation mechanisms by FtsZ and the other four proteins at the molecular level. To summarize our accomplishments with regard to minimal cell division, we have now successfully demonstrated the full cell-free reconstitution of a minimal division ring placement system, proving that such a complex biological feature can emerge from a set of well-defined components in vitro.

\section{Discussion}

In this work, we have reconstituted in vitro the bacterial division ring placement system from a minimal set of components, namely, MinC, MinD, MinE, FtsA, and FtsZ by cell-free expression. At the right choice of template ratios, proteins have been orchestrated reliably within giant lipid vesicles to form FtsZ-ring structures, and unexpectedly, even shown to constrict their membrane containers, despite being more than an order of magnitude larger than bacterial cells. In addition to the complete cell-free expressed system, we have quantitatively investigated the assembly and spatial regulation of a FtsZ-ring with MinCDE and FtsZ-Venus-mts in a fully controlled system based on purified proteins only, allowing us to evaluate the key factors involved in the process. Both approaches highlight important features of the bacterial division mechanism in vitro, confirming the relevance of the coupled protein systems FtsZ-FtsA and MinCDE as paradigms for minimal cell division in bottom-up synthetic biology.

One of the expected insights gained from our study is that MinCDE pole-to-pole oscillations indeed seem to be directly responsible for FtsZ-ring formation and placement in the sense that a previously isotropic mesh-like appearance of FtsZ filaments condensed into ring-like structures targeted to the equatorial region of the vesicles (Fig. 2c, d, 3d, and 5c). Interestingly, in some cases, we also confirmed that static bipolar localization of the Min proteins supports equatorial FtsZ-ring formation (Supplementary Fig. 6a and b). Not directly expected, but nevertheless highly plausible, was our finding that the condensation of FtsZ into ring-like structures, reminiscent of a spatial symmetry breaking, in turn led to a mode selection for oscillatory MinCDE dynamics in the sense that pole-to-pole oscillations were favored over pulsing or travelling wave modes in the presence of pronounced FtsZ-ring structures. Thus, Min self-organization dynamics and FtsZ-ring assembly appear to reinforce each other as a positive feedback mechanism towards bacterial cell division. Indeed, in most cases, FtsZ-ring structures and pole-to-pole oscillations persisted for a long time, supporting this hypothesis (Fig. 2d and Supplementary Movie 2). This positive feedback resulting in spatial symmetry breaking and establishment of a defined axis along which 
division is supposed to occur is particularly remarkable with respect with the originally spherical symmetry of our vesicle compartments, quite distinct from the rod-like shape of E.coli bacterial cells. In previous attempts of targeting circumferential actomyosin ring structures with contractile features to spherical membrane vesicles ${ }^{62}$, the lack of a defined division axis resulted in the slipping off from equatorial to polar regions upon ring constriction, in stark contrast of what can be observed in the presence of spatially stabilizing Min oscillations. We can thus conclude that any deviation from spherical symmetry leads to preferential mode selection of pole-to-pole oscillations.

In parallel to identify ideal biochemical conditions within cellular environments, we found that the likelihood of emerging Min pole-to-pole oscillations was only slightly increased under crowding conditions (Fig. 1d). However, in the presence of FtsZ, Min pole-to-pole oscillations appeared to increase with higher crowding conditions up to around $40 \%$ for Dextran70 at $100 \mathrm{~g} / \mathrm{L}$ (Fig. 2f), which coincides with a higher degree of FtsZ bundling under this condition (Supplementary Fig. 2c). Based on our findings of a positive feedback between the Min dynamics and FtsZ-ring structure formation, it is plausible to suggest that the enhanced formation of FtsZ bundles on the membrane at high crowding conditions is one of the key inducers of pole-to-pole oscillation mode selection. The latter agrees with the tight regulation of Min pole-to-pole oscillations in E. coli cells. Our observations point to a critical biological relevance of macromolecular crowding in the synergetic dynamics among Min waves, FtsZ-ring structures and environmental factors such as membrane interaction and confinement. We are only at the beginning of appreciating the mutual relationships of these factors to decipher their roles inside cells.

The other highlight of our results was the pronounced vesicle deformation induced by FtsZ-ring assembly and positioning in the complete set of cell-free expressed proteins. Even though there were previous reports on how FtsZ and related proteins may constrict or deform lipid vesicles ${ }^{16,23-26}$, our results allowed to visualize the gradual onset of force generation along with the condensation and positioning of a FtsZ-ring from an isotropic meshwork, thereby capturing the entire process of ring formation and vesicle constriction in a plausible chain of causation (Fig. 2d, 3d, and 5c). In agreement with our recent analysis, in which FtsZ-Venus-mts generates relatively weak forces that can only constrict deflated vesicles or wall-less E. coli cells ${ }^{26}$, we did not confirm any deformation of lipid vesicles by FtsZ-Venusmts rings under isosmotic conditions (Fig. 2d). In contrast, the FtsZ-ring composed of FtsZ-G55-VenusQ56 by anchoring through FtsA sufficiently constricted lipid vesicles even under isosmotic conditions (Fig. 5c). This suggests that the FtsZ-FtsA system might generate greater effective forces on membranes, potentially owing to a different mechanism of dynamic membrane attachment.

Moreover, we found a size dependence of vesicle deformation by FtsZ-rings (Fig. 5e and Supplementary Fig. 7), pointing to a higher degree of deformation in bigger vesicles. Since the surface-to-volume ratio becomes smaller in larger spheres, FtsZ-FtsA density on the membrane might be increased for larger vesicles at the same protein expression level, as shown in our analysis (Supplementary Fig. 4b and c, 5a and $b$ ). Therefore, higher density of FtsZ-FtsA on the membrane would explain why significant deformations can be only observed in larger vesicles, while lower membrane densities in smaller vesicles would not be able to induce major deformations. Besides, size-dependent mechanical properties of 
vesicle membrane such as surface tension and stiffness would favor higher deformation in larger vesicles $^{63-65}$. Biophysical quantitative measurements should be carried out in future studies to clarify the key features driving vesicle deformation among a broad range of vesicle sizes.

Additionally, our previous study showed that reversible membrane attachment of MinDE proteins through their amphipathic helices induces a spatiotemporal imbalance of membrane curvature ${ }^{41,66}$, and the same is expected to be true for FtsA. It is quite likely that in this dynamic system, periodic destabilization of the membrane aids large-scale transformation. In this regard, accumulation of transmembrane proteins that are also part of the bacterial divisome but have been missing in our system so far, such as ZipA, might enhance the local bending of the membrane ${ }^{67}$. Since the observed constriction of the initially spherical vesicles is still far away from a full scission process as in cell division, the influence of additional factors on membrane transformation will have to be further explored. ZipA, in particular, will be one of the most interesting next candidate proteins to improve the reconstitution of bacterial cell division.

In conclusion, although we successfully reconstituted the bacterial division ring placement system in vitro, and could even witness ring-induced shape transformation of the vesicles, further investigations are necessary to develop synthetic cell models that are truly able to autonomously self-divide in an energydissipating manner based on their intrinsic biological machinery. The most notorious challenge will presumably be the orchestration of division ring assembly and disassembly in the final process of membrane abscission. Although the exact sequence of events in bacteria is not fully clear, there is a high plausibility for a major mechanical role of cell wall expansion in vivo, a feature that presumably needs to be replaced by a more simplistic mechanism in minimal systems. However, one of the most promising candidates for additional large-scale force-inducing elements to aid FtsZ-ring constriction is actually the nucleoid itself, the transformation of which in the process of genome replication and segregation could easily couple to membrane transformation via biochemical, but also biophysical mechanisms, such as wetting and liquid phase separation ${ }^{68,69}$. Thus, moving from the reconstitution of a minimal division machinery, that has now been accomplished to a large extent, to a more holistic system which also comprises minimal genome replication, appears to be the next great goal of bottom-up synthetic biology.

\section{Methods}

\section{Plasmid construction}

Plasmids for protein purification and cell-free expression were constructed by seamless cloning or blunt end cloning method according to the provider's protocol. All enzymes for cloning were purchased from Thermo Fisher Scientific (Waltham, MA, USA). Briefly, DNA fragments were amplified with overlaps regions between adjacent fragments by PCR using Phusion High-Fidelity DNA Polymerase and origo primers (Sigma-Aldrich, St. Louis, MO, USA). Then, PCR products were treated with Dpnl and combined using GeneArt Seamless Cloning and Assembly Enzyme Mix. For deletion of sequences, a DNA fragment amplified from the original plasmid was treated with Dpnl, phosphorylated using T4 Phosphokinase, and then ligated with T4 DNA Ligase. All plasmids were propagated in E. coli OneShot TOP10 (Thermo Fisher 
Scientific) and purified from overnight culture using NucleoBond Xtra Midi kit (Macherey-Nagel GmbH, Duren, Germany). Constructed gene sequences were verified using Sanger Sequencing Service (Microsynth AG, Balgach, Switzerland). Detailed construction methods are described in Supplementary Methods, and all primers are listed in Supplementary Table 1.

\section{Protein purification}

His-MinD ${ }^{15}$, MinE-His ${ }^{15}$, FtsA $^{52}$, FtsZ-Venus-mts ${ }^{48}$ were purified and labeled as previously reported. Wildtype FtsZ was purified by calcium-induced precipitation ${ }^{70}$ and covalently labeled in the amino groups of $\mathrm{N}$-term amino acid residue with Alexa Fluor 488 carboxylic acid succinimidyl ester dye (Thermo Fisher Scientific) as earlier stated ${ }^{43}$.msfGFP-MinC, mCherry-MinC, mScarlet-I-MinC, and sfGFP were purified as described previously ${ }^{15,21}$. In brief, E. coli BL21 (DE3) pLysS cells were transformed with pET28a-HismsfGFP-MinC, pET28a-His-mCherry-MinC, pET28a-His-mScarlet-I-MinC, or pIVEX2.3d-sfGFP-His and incubated in LB medium (with $50 \mu \mathrm{g} / \mathrm{mL}$ Kanamycin or $100 \mu \mathrm{g} / \mathrm{mL}$ Ampicillin) at $37^{\circ} \mathrm{C}$ while shaking at $180 \mathrm{rpm}$. After an optical density at $600 \mathrm{~nm}$ reached $0.2-0.3$, Isopropyl-B-D-thiogalactopyranoside was added at a final concentration of $1 \mathrm{mM}$ to induce expression of the target protein. Cells were further cultured for 2-4 $\mathrm{h}$ and harvested. Consequently, cells were resuspended in Lysis buffer $(50 \mathrm{mM} \mathrm{Tris-HCl}$, $\mathrm{pH}$ 7.5, $300 \mathrm{mM} \mathrm{NaCl}, 10 \mathrm{mM}$ Imidazole) and disrupted using a tip sonicator (Branson ultrasonics S250D, Thermo Fisher Scientific). The crude cell lysate was separated by centrifugation for $30 \mathrm{~min}$ at $20,000 \times \mathrm{g}$ at $4{ }^{\circ} \mathrm{C}$, and the supernatant was mixed with Ni-NTA agarose (QIAGEN, Hilden, Germany). The sample was incubated for $10 \mathrm{~min}$ at $4{ }^{\circ} \mathrm{C}$ while gently shaking and loaded into an empty column. Ni-NTA agarose was washed with Wash buffer (50 mM Tris-HCl, pH 7.5, $300 \mathrm{mM} \mathrm{NaCl}, 20 \mathrm{mM}$ Imidazole, $10 \%$ Glycerol), and then the target protein was eluted with Elution buffer (50 mM Tris- $\mathrm{HCl}, \mathrm{pH} 7.5,300 \mathrm{mM}$ $\mathrm{NaCl}, 250 \mathrm{mM}$ Imidazole, $10 \%$ Glycerol). The buffer of the protein solution was exchanged with Storage buffer (50 mM Tris-HCl, pH 7.5, 150 mM GluK, 5 mM GluMg, 10 \% Glycerol) using Amicon Ultra-0.5 centrifugal filter unit $10 \mathrm{kDa}$ (Merck KGaA, Darmstadt, Germany).

FtsZ-G55-Venus-Q56 was purified using a calcium-induced precipitation method similar as described previously ${ }^{70}$. E. coli BL21 (DE3) pLysS cells were transformed with pET11b-FtsZ-G55-Venus-Q56, and the cell culture was prepared using LB medium (with $100 \mu \mathrm{g} / \mathrm{mL}$ Ampicillin) as described above. After collecting cell culture, the pellet was resuspended in PEM buffer (50 mM PIPES-NaOH, pH 6.5, $5 \mathrm{mM}$ $\mathrm{MgCl}_{2}, 1 \mathrm{mM}$ EDTA) and disrupted using a tip sonicator. The crude cell lysate was separated by centrifugation for $30 \mathrm{~min}$ at $20,000 \times \mathrm{g}$ at $4{ }^{\circ} \mathrm{C}$, and the supernatant was collected. The supernatant was mixed with $1 \mathrm{mM} \mathrm{GTP}$ and $20 \mathrm{mM} \mathrm{CaCl}_{2}$ and incubated at $30^{\circ} \mathrm{C}$ for 15 min to induce FtsZ bundles. Subsequently, the FtsZ bundles were pelleted by centrifugation for $15 \mathrm{~min}$ at $20,000 \times \mathrm{g}$ at $4{ }^{\circ} \mathrm{C}$, and the supernatant was discarded. The pellet was again resuspended in PEM buffer, and the supernatant was collected after a centrifugation for $15 \mathrm{~min}$ at $20,000 \times \mathrm{g}, 4^{\circ} \mathrm{C}$. Then, precipitation and resuspension steps were repeated (total two precipitation-resuspension cycles) to purify further. The buffer of the protein solution was exchanged with Storage buffer using Amicon Ultra- 0.5 centrifugal filter unit 50 kDa (Merck $\mathrm{KGaA})$. 
The concentration of the proteins was measured by Bradford Assay (Bio-Rad, Hercules, CA, USA), and protein solutions were aliquoted, frozen in liquid nitrogen, and stored at $-80^{\circ} \mathrm{C}$ until further use.

\section{Preparation of crowding solution}

Lyophilized BSA (Sigma-Aldrich, catalog number: A6003) was dissolved in Reaction buffer (50 mM Tris$\mathrm{HCl}, \mathrm{pH} 7.5,150 \mathrm{mM}$ GluK, $5 \mathrm{mM}$ GluMg) at approximately $100 \mathrm{~g} / \mathrm{L}$. Then, the buffer was exchanged with Reaction buffer to wash out residual molecules using Amicon Ultra-0.5 centrifugal filter unit $50 \mathrm{kDa}$. After washing steps, the concentration of BSA was measured by Bradford Assay. Ficoll70 and Dextran70 (Sigma-Aldrich) were dissolved in Reaction buffer, and their concentration was calculated from the weight of crowding agents and total volume of the solution (typically, the final concentration of the solution was $300-500 \mathrm{~g} / \mathrm{L})$. Crowding solutions were stored at $-20^{\circ} \mathrm{C}$ until further use.

\section{Preparation of lipid vesicles}

1-palmitoyl-2-oleoyl-glycero-3-phosphocholine (POPC) and 1-palmitoyl-2-oleoyl-sn-glycero-3-phospho-(1'rac-glycerol) (POPG) (Avanti Polar Lipids, Alabaster, AL, USA) were mixed at 7:3 molecular ratio dissolved in chloroform at $25 \mathrm{~g} / \mathrm{L}$. In case visualizing the lipid membrane, $2.5 \mathrm{mg} / \mathrm{L}$ of ATT0655 labeled 1,2dioleoyl-sn-glycero-3-phosphoethanolamine (DOPE) (ATTO-Tech GmbH, Siegen, Germany) was further mixed into the lipid mixture. $50 \mu \mathrm{L}$ of the lipid mixture was dried by nitrogen gas stream. $10 \mu \mathrm{L}$ of decane (TCl Deutschland GmbH, Eschborn, Germany) was added to the lipid film, and lipids were resuspended by vortexing shortly, and subsequently, $500 \mu \mathrm{L}$ of mineral oil (Carl Roth $\mathrm{GmbH}$, Karlsruhe, Germany) was added and vortexed for 1 min to prepare a lipid-oil mixture.

We used the double emulsion transfer method for vesicle production to yield giant unilamellar vesicles $^{33,40}$. In case reconstitution with purified proteins, both inner and outer solutions were prepared in Reaction buffer. For cell-free expression experiments, a homemade PURE solution I based on a previous report ${ }^{54}$ was used as the outer solution. The osmolarity of inner and outer was measured using an osmometer (Fiske Micro-Osmometer model120, Fiske Associates, Norwood, MA, USA), and outer buffer was diluted to match the osmolarities between inner and outer solution.

Lipid vesicles containing purified proteins or cell-free expression systems were formed following the same methodology with slight differences. For the case of purified proteins, a 96-Well Flat-Bottom Microplate (SensoPlate, Greiner Bio-One $\mathrm{GmbH}$, Kremsmuenster, Austria) was used for vesicle formation and visualization, while for the cell-free system, vesicles were formed using a $1.5 \mathrm{~mL}$ tube. For the encapsulation of purified proteins, emulsion solution was obtained from $2 \mu \mathrm{l}$ of the inner solution and $100 \mu \mathrm{L}$ of the lipid-oil mixture mixed in a $1.5 \mathrm{~mL}$ tube by tapping. $100 \mu \mathrm{L}$ of the outer solution was added into a well in a 96 well-plate, and subsequently, $50 \mu \mathrm{L}$ of the lipid-oil mixture was layered on the outer solution. Then, $\sim 80 \mu \mathrm{L}$ of the emulsion solution was further dripped on the multi-layered solution. For the encapsulation of the Cell-free system, emulsion solution was formed by $5 \mu$ of the inner solution and 250 $\mu \mathrm{L}$ of the lipid-oil mixture in a $1.5 \mathrm{~mL}$ tube. $500 \mathrm{uL}$ of the outer solution was added to a fresh $1.5 \mathrm{~mL}$ tube, 
and subsequently, $200 \mathrm{uL}$ of the lipid-oil mixture was layered to form a lipid monolayer. $200 \mathrm{uL}$ of the emulsion was added carefully to the multi-layered solution.

After multi-layering, lipid vesicles were obtained by centrifugation for $10 \mathrm{~min}$ at room temperature (for purified proteins) or $4^{\circ} \mathrm{C}$ (for cell-free expression) at appropriate centrifugation force. Applied centrifugation force was varied depending on the density of the inner solution given by crowding agents. Typically, we used $3,000 \times \mathrm{g}$ for $10 \mathrm{~g} / \mathrm{L}, 500 \times \mathrm{g}$ for $60 \mathrm{~g} / \mathrm{L}$, and $300 \mathrm{xg}$ for $110 \mathrm{~g} / \mathrm{L}$ of the density of the inner solution. After centrifugation, the oil phase and the supernatant of the water phase were discarded. The rest of the water phase (approximately $100 \mu \mathrm{L}$ ) was then gently mixed by pipetting to resuspend lipid vesicles, and $50 \mu \mathrm{L}$ of the vesicle solution was restored in another fresh tube.

\section{Self-organization assays inside lipid vesicles with purified protein}

For reconstitution of the MinCDE system, an inner solution containing different concentrations of MinD and MinE $(0.25-3 \mu \mathrm{M})$ and $0.5 \mu \mathrm{M}$ mScarlet-MinC or mCherry MinC were mixed with $10 \mathrm{mg} / \mathrm{ml} \mathrm{BSA}$ and $2.5 \mathrm{mM}$ ATP in Reaction buffer. For the co-reconstitution of FtsZ and Min system, FtsZ-Venus-mts was added to this mixture at $2 \mu \mathrm{M}$ in addition to $2 \mathrm{mM}$ GTP to trigger its polymerization. In samples containing crowder, different concentration of either Ficoll70 or Dextran70 was added at $10-100 \mathrm{~g} / \mathrm{L}$ as the final concentration. Assays using only FtsZ were carried out following the same methodology in the absence of the Min system and ATP. All proteins and crowders were previously dialyzed or diluted in Reaction buffer.

\section{Cell-free expression inside lipid vesicles}

Cell-free expression was carried out using PUREfrex 2.0 (GeneFrontier, Chiba, Japan) following the supplier's instruction. DNA templates were linearized from corresponding plasmids using PrimeSTAR Max DNA polymerase (Takara Bio, Shiga, Japan) with T7P-F and T7P-R primers (Supplementary Table 1). Linearized templates and original templates are listed in Supplementary Table 2. The inner solution consisted of PUREfrex 2.0, linearized DNA templates, $10 \mathrm{~g} / \mathrm{L} \mathrm{BSA}$, and $50 \mathrm{~g} / \mathrm{L}$ Ficoll70. For expression with purified proteins, $2 \mu \mathrm{M}$ FtsZ-Alexa488, $0.5 \mu \mathrm{M}$ mCherry-MinC, or $0.5 \mu \mathrm{M}$ msfGFP-MinC were additionally supplied to the inner solution. The concentration of DNA templates and ratios were optimized to obtain the Min waves patterns and FtsZ structures. All template conditions are listed in Supplementary Table 3. The crowding agent and its concentration were varied for the estimation of sfGFP expression yield.

A homemade chamber was prepared for incubation with the temperature control stage. Coverslips (Menzel Glasses, \# 1.5, $22 \mathrm{~mm} \times 22 \mathrm{~mm}$, Thermo Fisher Scientific) were washed with 70\% EtOH. Then, three imaging spacers (Grace Bio-Labs SecureSeal imaging spacers, 1 well, $9 \mathrm{~mm} \times 0.12 \mathrm{~mm}$, SigmaAldrich) were stacked on a glass slip. $20 \mu \mathrm{L}$ of $10 \mathrm{~g} / \mathrm{BSA}$ solution was added to the camber to passivate the glass surface and leave for $10 \mathrm{~min}$ at room temperature, and subsequently, BSA solution was discarded and then washed with outer buffer. $30 \mathrm{uL}$ of vesicle solution was added to the homemade chamber, and the chamber was enclosed by another coverslip and sealed. Then, the chamber was 
mounted to the PE120-XY Peltier system (Linkam Scientific Instruments, Surrey, United Kingdom) and placed on Zeiss LSM780 confocal laser scanning microscope (Carl Zeiss AG, Oberkochen, Germany). The temperature was set at $4{ }^{\circ} \mathrm{C}$ before observation and then kept at $37^{\circ} \mathrm{C}$ for cell-free expression.

\section{Microscopy and image processing}

Images of lipid vesicle samples were taken by a Zeiss LSM780 confocal laser scanning microscope using a Plan-Apochromat 20x/0.80 air objective or C-Apochromat 40x/1.20 water-immersion objective (Carl Zeiss AG). Fluorophores were excited using a $488 \mathrm{~nm}$ Argon laser (for Alexa488, sfGFP, msfGFP, and Venus), $561 \mathrm{~nm}$ diode-pumped solid-state laser (for mCherry and mScarlet-l), and $633 \mathrm{~nm} \mathrm{He}-\mathrm{Ne}$ laser (for ATT0655). Images were typically acquired with 512 x 512 pixel resolution, 10-15 Z-stacks with 1-2 $\mu \mathrm{m}$ intervals, and 15-20 s intervals for $10 \mathrm{~min}$ to $3 \mathrm{~h}$. All recorded tiff images were processed, visualized, and analyzed using Fiji ${ }^{71}$ (v1.53f). Z-stacks were visualized in 3D max reconstituted images by the Z projection function. Kymographs were generated using a custom ImageJ macro script. In short, peripheral of the vesicles were detected from manually drawn ROIs, and then straightened to obtain time-stacked straight-line images. Subsequently, lines were stacked into an orthogonal direction against the long axis of the images in order of elapsed time (Fig. 1b).

\section{Self-organization assay on SLBs}

Preparation of the supported lipid bilayers (SLBs) was described previously ${ }^{30}$. Briefly, a plastic chamber was attached to a cleaned glass coverslip (Menzel Glasses) using ultraviolet-curable glue (Norland Optical Adhesive 63, Norland Products Inc., Jamesburg, NJ, USA). The slide was cleaned in an oxygen plasma cleaner (model Zepto, Diener electronic, Ebhausen, Germany) for 30 seconds at $50 \%$ power. Small unilamellar vesicles (SUVs) were prepared at a concentration of $4 \mathrm{mg} / \mathrm{ml}$ of 1,2-dioleoyl-sn-glycero-3phosphocholine (DOPC) and 1,2-dioleoyl-sn-glycero-3-phospho-(1'-rac-glycerol) (DOPG) mixture (DOPC:DOPG = 7:3 molar ratio) in a buffer ( $25 \mathrm{mM}$ Tris- $\mathrm{HCl} \mathrm{pH} \mathrm{7.5,150} \mathrm{mM} \mathrm{KCl,} 5 \mathrm{mM} \mathrm{MgCl} 2$ ) by sonication in a sonicator bath. To generate the SLBs, SUVs were added to the reaction chamber at a concentration of $0.5 \mathrm{mg} / \mathrm{ml}$ and incubated for $3 \mathrm{~min}$ on a $37^{\circ} \mathrm{C}$ warm heating block. The SLBs were washed 10 times with a total of $2 \mathrm{ml}$ in the same buffer in the absence of magnesium ( $50 \mathrm{mM}$ Tris-HCl $\mathrm{pH} 7.5,150 \mathrm{mM} \mathrm{KCl}$ ) to remove excess vesicles.

Before self-organization assays, the buffer in the chamber was exchanged with Reaction buffer. Then, FtsA was added to the Reaction buffer at $0.25 \mu \mathrm{M}$. After $\sim 2$ minutes of incubation, a mixture of either FtsZ-wt and FtsZ-Alexa488 (30\%) or FtsZ-G55-Venus-Q56 at $1 \mu \mathrm{M}$ was added to the chamber.

Subsequently, $1 \mathrm{mM}$ ATP and $1 \mathrm{mM}$ GTP were added and mixed carefully. SLBs were incubated for $>10$ minutes before visualization. Fluorescence imaging was carried out on an inverted custom-built TIRF microscope ${ }^{72}$ with a UAPON 100x/1.49 oil-immersion objective (Olympus, Tokyo, Japan). Excitation of the sample was made using a $488 \mathrm{~nm}$ laser, and the fluorescence signal was detected on a CMOS camera (Zyla 4.2, Andor Technology, Belfast, Northern Ireland).

\section{GTPase assay of FtsZ}


GTPase activity of FtsZ was measured by quantifying the inorganic phosphate with a colorimetric phosphate quantification assay (BIOMOL GREEN kit, ENZO life sciences, Lörrach, Germany) for 140 seconds ${ }^{49}$. Purified FtsZ-wt or FtsZ-G55-Venus-Q56 were used at $3 \mu \mathrm{M}$ in Reaction buffer, and polymerization was triggered by $1 \mathrm{mM} \mathrm{GTP.} 13 \mathrm{uL}$ fractions were added to a 96-Well Flat-Bottom Microplate (UV-Star, Greiner Bio-One GmbH) every $20 \mathrm{sec}$ after addition of GTP and mixed with $37 \mathrm{uL}$ of Reaction buffer and $100 \mathrm{uL}$ of BIOMOL GREEN reagent, stopping the reaction. After $\sim 10 \mathrm{~min}$ of incubation at RT, the absorbance at $620 \mathrm{~nm}$ was measured in a TECAN plate reader (Tecan Group Ltd., Mannedorf, Switzerland) at room temperature. Phosphate concentrations were calculated from a $\mathrm{Na}_{2} \mathrm{HPO}_{4}$ standard curve in Reaction buffer, and the GTPase activity reaction rate $(\mathrm{V}, \mathrm{mol} \mathrm{Pi} / \mathrm{mol}$ FtsZ/min) was determined from the slope of the linear part of phosphate accumulation curves.

\section{Analysis of the wave patterns and FtsZ structures inside lipid vesicles}

Wave patterns of MinCDE proteins inside vesicles were analyzed using a custom ImageJ macro script. Briefly, time-lapse images were used to detect the lipid vesicles and obtain a kymograph from the fluorescence intensity on the peripheral membrane. These Kymographs were classified and checked manually in order to calculate the appearance ratios of each wave mode at each condition. FtsZ structures were visualized and classified manually using the 3D-max of the lipid vesicles containing FtsZ.

\section{Estimation of the sfGFP expression level within lipid vesicles}

First, different concentration series of purified $\operatorname{sfGFP}(0,0.1,0.3,1,3,10$, or $30 \mu \mathrm{M})$ was encapsulated in vesicles, and the fluorescence intensity of sfGFP was measured ( $n=30$ individual vesicles) from confocal images for each concentration to obtain the standard curve. Then, sfGFP was expressed inside lipid vesicles with different macromolecular crowding conditions (no additives, $50 \mathrm{~g} / \mathrm{L}$ Ficoll70, $100 \mathrm{~g} / \mathrm{L}$ Ficoll70, $50 \mathrm{~g} / \mathrm{L}$ Dextran70, or $100 \mathrm{~g} / \mathrm{L}$ Dextran70), and the fluorescence intensity of sfGFP was measured within 100 individual vesicles for $200 \mathrm{~min}$ with $1 \mathrm{~min}$ intervals. The box plots were obtained from fluorescence intensities at 200 min (for Fig. 3b), and time-development of sfGFP expression level was calculated from fluorescence intensities at each time point (Supplementary Fig. 3b-f).

\section{Analysis of the FtsZ localization inside lipid vesicles along with FtsA expression}

Cell-free expression of FtsA was performed with $2 \mu \mathrm{M}$ FtsZ-Alexa488 ( $f t s A$ template was omitted for a negative control) for 60 min with $20 \mathrm{sec}$ intervals (total 180 time points, recording started after 20 min incubation at $37^{\circ} \mathrm{C}$ ). Fluorescence intensity of FtsZ-Alexa488 in the lumen or membrane at the equatorial plane was then measured with a representative vesicle and average intensities at each time point were plotted in Fig. 3c or Supplementary Fig. 4b. The same procedure was repeated to measure the intensity of FtrsZ-G55-Venus-Q56 on membrane in the co-expression experiment of FtsA/FtrsZ-G55-Venus-Q56 and plotted in Supplementary Fig. 5a. In both cases, average fluorescence intensities of FtsZ on membrane among different vesicles were measured after cell-free expression (total $80 \mathrm{~min}$ ) and plotted against vesicle diameter in Supplementary Fig. 4c and 5b. 


\section{Estimation of the wave occurrence with cell-free expressed Min proteins}

Cell-free co-expression of MinD/MinE or mCherry-MinC/MinD/MinE was performed with $0.5 \mu \mathrm{M}$ purified msfGFP-MinC for 50 min with $20 \mathrm{sec}$ intervals (total 150 time points, recording started after 10 min incubation at $\left.37^{\circ} \mathrm{C}\right)$. Then, vesicles were randomly chosen $(n=116$ and 100 for MinDE and MinCDE expression, respectively.) and the percentage of Min wave patterns inside lipid vesicles were calculated as (sum of the dynamic waves and static patterns)/(vesicle number) at every $3 \mathrm{~min}$ time point and plotted in Fig. 4c.

\section{Analysis of FtsZ structures and the aspect ratio of deformed vesicles}

Before and after 2-3 h of co-expression of FtsA/FtrsZ-G55-Venus-Q56/mCherry-MinC/MinD/MinE, Z-stack images of Venus fluorescence were acquired with tile-scan function. Vesicles were classified into four size ranges $(8-12,12-16,16-20,>20 \mu \mathrm{m}$ in a diameter, $\mathrm{n}=40$ for each range) from the images before expression. The structures of FtsZ were then detected manually from 3D max projection images, and vesicles with FtsZ-ring structure were further analyzed to determine the aspect ratio. The aspect ratio of the deformed vesicles was calculated as (length of short axis)/(length of long axis). Also, the degree of deformation was calculated as (length of short axis)/(diameter of the vesicle before deformation). The status of the vesicles was classified into three (no ring structure, ring structure, ring structure + deformation (with $<0.95$ aspect ratio)) and plotted in Fig. $5 \mathrm{~d}$. The relation of the aspect ratio of the vesicles with FtsZ-ring and the original vesicle sizes were further plotted in Fig. 5e, and the correlation coefficient was calculated among all vesicles plotted in the figure $(n=79)$.

\section{Surface plot of the FtsZ distribution inside a lipid vesicle}

The kymograph of FtsZ distribution at an equatorial plane of the vesicle shown in Supplementary Fig. 6b was obtained using a custom ImageJ macro. Then, fluorescence intensities of the FtsZ were measured at every 10 min from the kymograph. The intensity profiles were then plotted against relative position on the peripheral of the vesicle $\left(0^{\circ}-360^{\circ}\right)$ in Supplementary Fig. $6 \mathrm{~d}$.

\section{Declarations}

\section{Data availability}

All data supporting the findings of this manuscript and custom ImageJ macro scripts are available from the corresponding author upon reasonable request.

\section{Acknowledgments}

Special thanks to Germán Rivas (CIB Margarita Salas, CSIC, Spain) for providing essential scientifiC advice on the self-organization with purified proteins under crowding, and his helpful comments and critical reviews on the manuscript. We are grateful to Lei Kai (School of Life Sciences, Jiangsu Normal University, China) for the plasmid pIVEX2.3d-sfGFP-His, Leon Harrington and Tamara Heermann for their 
help in plasmid construction. We would like to thank MPIB Core Facility for assistance in protein purification, Michaela Schaper for plasmid cloning, Katharina Nakel for GTPase assay, Sigrid Bauer for lipid preparation, Kerstin Andersson for protein purification, and Michele Russo for assistance with the purification of FtsZ-G55-Venus-Q56. We are also grateful to Jan-Hagen Krohn and Yusuf Qutbuddin for assistance in TIRF microscopy. Further, we thank Leon Babl, Hiromune Eto, Ana Yagüe Relimpio, Henri Franquelim, and Michele Russo for helpful discussions. This work has been supported by JSPS Overseas Research Fellowships (S.K) and the Max Planck-Bristol Centre for Minimal Biology (A.M.-S). A.M.-S is part of IMPRS-LS.

\section{Author contributions}

S.K., A.M.-S., and P.S. conceived the study and wrote the manuscript. S.K. designed, performed, and analyzed all experiments with cell-free expression. S.K. made the custom ImageJ macro script for the analysis and kymograph visualization. A.M.-S. designed, performed, and analyzed all experiments with purified proteins within vesicles and SLBs. S.K. designed plasmids for purification and expression of sfGFP, msfGFP-MinC, mScarlet-I-MinC, mCherry-MinC, FtsZ-Venus, and FtsZ-G55-Venus-Q56. S.K. also purified sfGFP, msfGFP-MinC, mScarlet-I-MinC, mCherry-MinC, and FtsZ-G55-Venus-Q56. A.M.-S. purified and labeled FtsZ-wt. A.M.-S. also characterized FtsZ-G55-Venus-Q56 and FtsZ-wt by performing a GTPase assay.

Competing interests: The authors declare no competing interests.

\section{References}

1. Schwille, P. Bottom-up synthetic biology: Engineering in a tinkerer's world. Science 333, 1252-1254 (2011).

2. Schwille, P. et al. MaxSynBio: Avenues towards creating cells from the bottom up. Angew. Chemie Int. Ed. 57, 13382-13392 (2018).

3. Rivas, G., Vogel, S. K. \& Schwille, P. Reconstitution of cytoskeletal protein assemblies for large-scale membrane transformation. Curr. Opin. Chem. Biol. 22, 18-26 (2014).

4. Mulla, Y., Aufderhorst-Roberts, A. \& Koenderink, G. H. Shaping up synthetic cells. Phys. Biol. 15, (2018).

5. Stano, P. Is research on "Synthetic cells" moving to the next level? Life 9, 3 (2018).

6. Rothfield, L., Justice, S. \& García-Lara, J. Bacterial cell division. Annu. Rev. Genet. 33, 423-448 (1999).

7. Scholey, J. M., Brust-Mascher, I. \& Mogilner, A. Cell division. Nature 422, 746-752 (2003).

8. Natale, P. \& Vicente, M. Bacterial cell division. eLS1-9 (2020). 
9. Loose, M., Kruse, K. \& Schwille, P. Protein self-organization: Lessons from the Min system. Annu. Rev. Biophys. 40, 315-336 (2011).

10. Ramm, B., Heermann, T. \& Schwille, P. The E. coli MinCDE system in the regulation of protein patterns and gradients. Cell. Mol. Life Sci. 76, 4245-4273 (2019).

11. Raskin, D. M. \& de Boer, P. A. J. Rapid pole-to-pole oscillation of a protein required for directing division to the middle of Escherichia coli. Proc. Natl. Acad. Sci. USA 96, 4971-4976 (1999).

12. Hu, Z. \& Lutkenhaus, J. Topological regulation of cell division in Escherichia coli involves rapid pole to pole oscillation of the division inhibitor MinC under the control of MinD and MinE. Mol. Microbiol. 34, 82-90 (1999).

13. Bi, E. \& Lutkenhaus, J. FtsZ ring structure associated with division in Escherichia coli. Nature 354, 161-164 (1991).

14. McQuillen, R. \& Xiao, J. Insights into the structure, function, and dynamics of the bacterial cytokinetic FtsZ-ring. Annual Review of Biophysics 49, 309-341 (2020).

15. Loose, M., Fischer-Friedrich, E., Ries, J., Kruse, K. \& Schwille, P. Spatial regulators for bacterial cell division self-organize into surface waves in vitro. Science 320, 789-792 (2008).

16. Osawa, M., Anderson, D. E. \& Erickson, H. P. Reconstitution of contractile FtsZ rings in liposomes. Science 320, 792-794 (2008).

17. Zhou, H.-X., Rivas, G. \& Minton, A. P. Macromolecular crowding and confinement: biochemical, biophysical, and potential physiological consequences. Annu. Rev. Biophys. 37, 375-397 (2008).

18. Van Den Berg, J., Boersma, A. J. \& Poolman, B. Microorganisms maintain crowding homeostasis. Nat. Rev. Microbiol. 15, 309-318 (2017).

19. Montes, L. R., Alonso, A., Goñi, F. M. \& Bagatolli, L. A. Giant unilamellar vesicles electroformed from native membranes and organic lipid mixtures under physiological conditions. Biophys. J. 93, 3548-3554 (2007).

20. Litschel, T. \& Schwille, P. Protein reconstitution inside giant unilamellar vesicles. Annual Review of Biophysics 50, 525-548 (2021).

21. Kohyama, S., Yoshinaga, N., Yanagisawa, M., Fujiwara, K. \& Doi, N. Cell-sized confinement controls generation and stability of a protein wave for spatiotemporal regulation in cells. eLife 8, e44591 (2019).

22. Kohyama, S., Fujiwara, K., Yoshinaga, N. \& Doi, N. Conformational equilibrium of MinE regulates the allowable concentration ranges of a protein wave for cell division. Nanoscale 12, 11960-11970 (2020). 
23. Osawa, M. \& Erickson, H. P. Liposome division by a simple bacterial division machinery. Proc. Natl. Acad. Sci. USA 110, 11000-11004 (2013).

24. Furusato, T. et al. De novo synthesis of basal bacterial cell division proteins FtsZ, FtsA, and ZipA inside giant vesicles. ACS Synth. Biol. 7, 953-961 (2018).

25. Godino, E. et al. Cell-free biogenesis of bacterial division proto-rings that can constrict liposomes. Commun. Biol. 3, 539 (2020).

26. Ramirez-Diaz, D. A. et al. FtsZ induces membrane deformations via torsional stress upon GTP hydrolysis. Nat. Commun. 12, 3310 (2021).

27. Ganzinger, K. A. et al. FtsZ reorganization facilitates deformation of giant vesicles in microfluidic traps. Angew. Chemie Int. Ed. 132, 21556-21560 (2020).

28. Martos, A. et al. FtsZ polymers tethered to the membrane by ZipA are susceptible to spatial regulation by Min waves. Biophys. J. 108, 2371-2383 (2015).

29. Zieske, K., Chwastek, G. \& Schwille, P. Protein patterns and oscillations on lipid monolayers and in microdroplets. Angew. Chemie Int. Ed. 55, 13455-13459 (2016).

30. Ramm, B. et al. The MinDE system is a generic spatial cue for membrane protein distribution in vitro. Nat. Commun. 9, 3942 (2018).

31. Godino, E. et al. De novo synthesized Min proteins drive oscillatory liposome deformation and regulate FtsA-FtsZ cytoskeletal patterns. Nat. Commun. 10, 4969 (2019).

32. Rivas, G., Alfonso, C., Jiménez, M., Monterroso, B. \& Zorrilla, S. Macromolecular interactions of the bacterial division FtsZ protein: From quantitative biochemistry and crowding to reconstructing minimal divisomes in the test tube. Biophys. Rev. 5, 63-77 (2013).

33. Yoshida, A., Kohyama, S., Fujiwara, K., Nishikawa, S. \& Doi, N. Regulation of spatiotemporal patterning in artificial cells by a defined protein expression system. Chem. Sci. 10, 11064-11072 (2019).

34. Glock, P. et al. Stationary patterns in a two-protein reaction-diffusion system. ACS Synth. Biol. 8, 148-157 (2018).

35. Yue, K., Zhu, Y. \& Kai, L. Cell-free protein synthesis: Chassis toward the minimal cell. Cells 8, 315 (2019).

36. Garenne, D. et al. Cell-free gene expression. Nat. Rev. Methods Prim. 1, 49 (2021).

37. Rueda, S., Vicente, M. \& Mingorance, J. Concentration and assembly of the division ring proteins FtsZ, FtsA, and ZipA during the Escherichia coli cell cycle. J. Bacteriol. 185, 3344-3351 (2003). 
38. Schmidt, A. et al. The quantitative and condition-dependent Escherichia coli proteome. Nat. Biotechnol. 34, 104-110 (2016).

39. Robles-Ramos, M. Á. et al. Assembly of bacterial cell division protein FtsZ into dynamic biomolecular condensates. Biochim. Biophys. Acta - Mol. Cell Res. 1868, 118986 (2021).

40. Pautot, S., Frisken, B. J. \& Weitz, D. A. Engineering asymmetric vesicles. Proc. Natl. Acad. Sci. USA 100, 10718-10721 (2003).

41. Litschel, T., Ramm, B., Maas, R., Heymann, M. \& Schwille, P. Beating vesicles: Encapsulated protein oscillations cause dynamic membrane deformations. Angew. Chemie Int. Ed. 57, 16286-16290 (2018).

42. Rivas, G., Fernández, J. A. \& Minton, A. P. Direct observation of the enhancement of noncooperative protein self-assembly by macromolecular crowding: indefinite linear self-association of bacterial cell division protein FtsZ. Proc. Natl. Acad. Sci. USA 98, 3150-3155 (2001).

43. González, J. M. et al. Essential cell division protein FtsZ assembles into one monomer-thick ribbons under conditions resembling the crowded intracellular environment. J. Biol. Chem. 278, 3766437671 (2003).

44. Groen, J. et al. Associative interactions in crowded solutions of biopolymers counteract depletion effects. J. Am. Chem. Soc. 137, 13041-13048 (2015).

45. Rivas, G. \& Minton, A. P. Macromolecular crowding in vitro, in vivo, and in between. Trends Biochem. Sci. 41, 970-981 (2016).

46. Norred, S. E. et al. Macromolecular crowding induces spatial correlations that control gene expression bursting patterns. ACS Synth. Biol. 7, 1251-1258 (2018).

47. Cabré, E. J. et al. Bacterial division proteins FtsZ and ZipA induce vesicle shrinkage and cell membrane invagination. J. Biol. Chem. 288, 26625-26634 (2013).

48. Ramirez-Diaz, D. A. et al. Treadmilling analysis reveals new insights into dynamic FtsZ ring architecture. PLOS Biol. 16, e2004845 (2018).

49. García-Soriano, D. A., Heermann, T., Raso, A., Rivas, G. \& Schwille, P. The speed of FtsZ treadmilling is tightly regulated by membrane binding. Sci. Rep. 10, 10447 (2020).

50. Martos, A., Jiménez, M., Rivas, G. \& Schwille, P. Towards a bottom-up reconstitution of bacterial cell division. Trends Cell Biol. 22, 634-643 (2012).

51. Hale, C. A. \& De Boer, P. A. J. Direct binding of FtsZ to ZipA, an essential component of the septal ring structure that mediates cell division in E. coli. Cel/ 88, 175-185 (1997). 
52. Loose, M. \& Mitchison, T. J. The bacterial cell division proteins ftsA and ftsZ self-organize into dynamic cytoskeletal patterns. Nat. Cell Biol. 16, 38-46 (2014).

53. Shimizu, Y. et al. Cell-free translation reconstituted with purified components. Nat. Biotechnol. 19, 751-755 (2001).

54. Kuruma, Y. \& Ueda, T. The PURE system for the cell-free synthesis of membrane proteins. Nat. Protoc. 10, 1328-1344 (2015).

55. Ge, X., Luo, D. \& Xu, J. Cell-free protein expression under macromolecular crowding conditions. PLoS One 6, e28707 (2011).

56. Takahashi, K., Sato, G., Doi, N. \& Fujiwara, K. A relationship between ntp and cell extract concentration for cell-free protein expression. Life 11, 237 (2021).

57. Ma, X., Ehrhardt, D. W. \& Margolin, W. Colocalization of cell division proteins FtsZ and FtsA to cytoskeletal structures in living Escherichia coli cells by using green fluorescent protein. Proc. Nat. Acad. Sci. USA 93, 12998-13003 (1996).

58. Osawa, M. \& Erickson, H. P. Probing the domain structure of FtsZ by random truncation and insertion of GFP. Microbiology 151, 4033-4043 (2005).

59. Buske, P. J. \& Levin, P. A. A flexible C-terminal linker is required for proper FtsZ assembly in vitro and cytokinetic ring formation in vivo. Mol. Microbiol. 89, 249-263 (2013).

60. Ortiz, C., Natale, P., Cueto, L. \& Vicente, M. The keepers of the ring: Regulators of FtsZ assembly. FEMS Microbiol. Rev. 40, 57-67 (2015).

61. Moore, D. A., Whatley, Z. N., Joshi, C. P., Osawa, M. \& Erickson, H. P. Probing for binding regions of the FtsZ protein surface through site-directed insertions: Discovery of fully functional FtsZ-fluorescent proteins. J. Bacteriol. 199, e00553-16 (2017).

62. Litschel, T. et al. Reconstitution of contractile actomyosin rings in vesicles. Nat. Commun. 12, (2021).

63. Lin, C. M., Li, C. S., Sheng, Y. J., Wu, D. T. \& Tsao, H. K. Size-dependent properties of small unilamellar vesicles formed by model lipids. Langmuir 28, 689-700 (2012).

64. Lipowsky, R. Remodeling of membrane shape and topology by curvature elasticity and membrane tension. Adv. Biol. 6, 2101020 (2021).

65. Dimova, R. \& Marques, C. The Giant Vesicle Book. (CRC Press, 2019).

66. Christ, S., Litschel, T., Schwille, P. \& Lipowsky, R. Active shape oscillations of giant vesicles with cyclic closure and opening of membrane necks. Soft Matter 17, 319-330 (2021). 
67. Derganc, J. \& Čopič, A. Membrane bending by protein crowding is affected by protein lateral confinement. Biochim. Biophys. Acta - Biomembr. 1858, 1152-1159 (2016).

68. Li, Y., Lipowsky, R. \& Dimova, R. Transition from complete to partial wetting within membrane compartments. J. Am. Chem. Soc. 130, 12252-12253 (2008).

69. Andes-Koback, M. \& Keating, C. D. Complete budding and asymmetric division of primitive model cells to produce daughter vesicles with different interior and membrane compositions. J. Am. Chem. Soc. 133, 9545-9555 (2011).

70. Rivas, G. et al. Magnesium-induced linear self-association of the FtsZ bacterial cell division protein monomer. The primary steps for FtsZ assembly. J. Biol. Chem. 275, 11740-11749 (2000).

71. Schindelin, J. et al. Fiji: An open-source platform for biological-image analysis. Nat. Methods 9 , 676-682 (2012).

72. Stehr, F., Stein, J., Schueder, F., Schwille, P. \& Jungmann, R. Flat-top TIRF illumination boosts DNAPAINT imaging and quantification. Nat. Commun. 10, 1268 (2019).

\section{Figures}

a

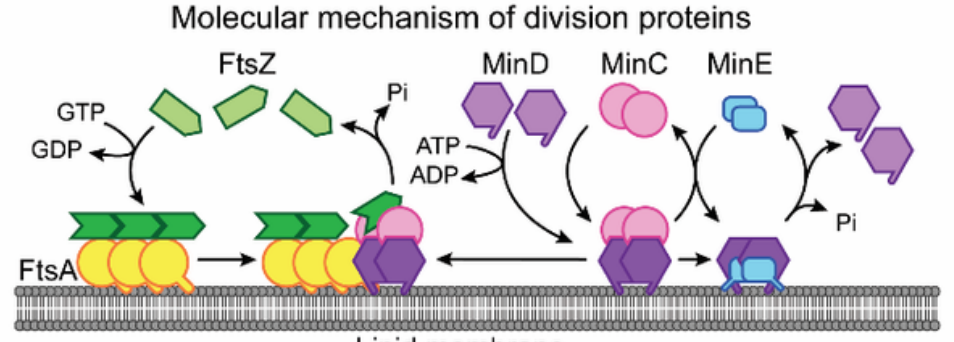

Lipid membrane

b
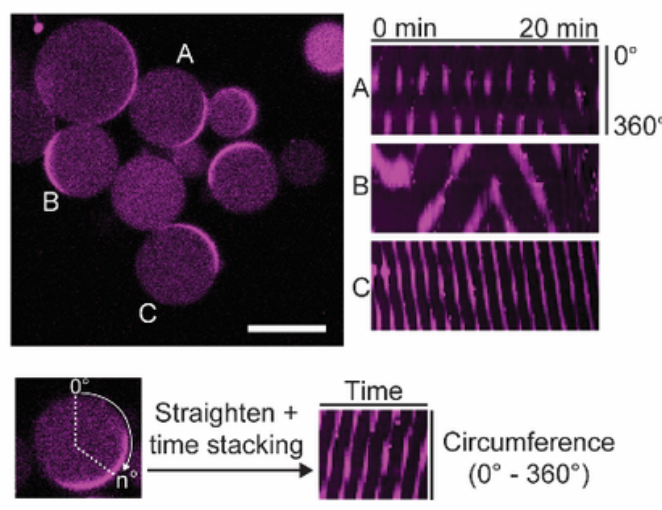

Straighten +
C

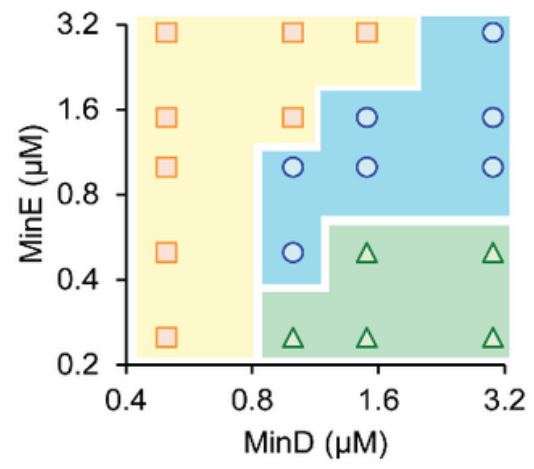

ODynamic waves $\square$ lumen $\Delta$ Membrane

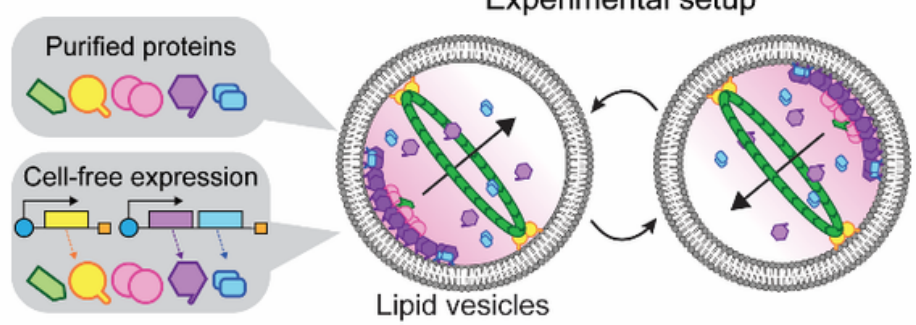

d

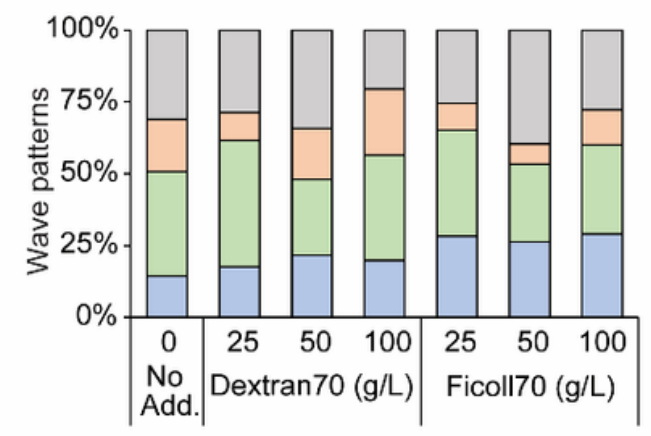

$\square$ No waves $\square$ Pulsing

$\square$ Traveling $\square$ Pole-to-pole

\section{Figure 1}

Characterization of MinCDE dynamics under different macromolecular crowding conditions. a Schematic illustration of the MinCDE/FtsZ-FtsA self-organization system (left) introduced into vesicles (right). B 
Left: Representative confocal images of purified MinCDE proteins inside lipid vesicles in the presence of macromolecular crowding. Capital alphabet letters correspond to the kymographs on the right side. Scale bar $15 \mu \mathrm{m}$. Right: Kymographs of different MinCDE wave patterns captured by mScarlet-I-MinC

fluorescence. C Phase diagram of major MinCDE pattern formation inside lipid vesicles without crowding conditions (Dynamic waves, localization in lumen, and localization on membrane, vs. MinDE concentrations). D Variation of MinCDE dynamics (absence of waves, pole-to-pole oscillations, traveling waves, or pulsing) inside vesicles at different macromolecular crowding concentrations (0-100 g/L) using Dextran70 or Ficoll70. ( $\mathrm{n}=374,227,181,161,181,298,303$ for no additives, 25, 50, $100 \mathrm{~g} / \mathrm{L}$ Dextran70, $25,50,100 \mathrm{~g} / \mathrm{L}$ Ficoll70, respectively).

a

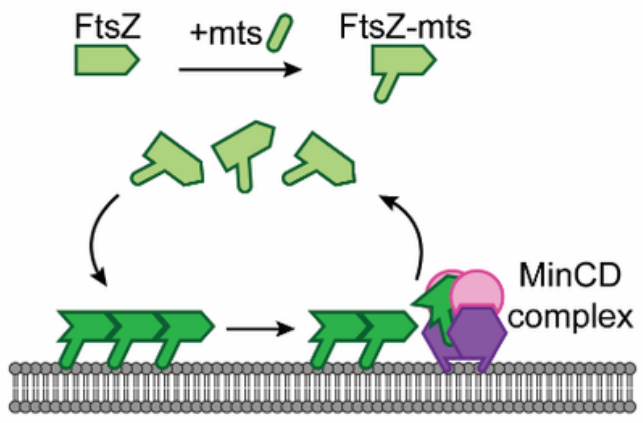

b

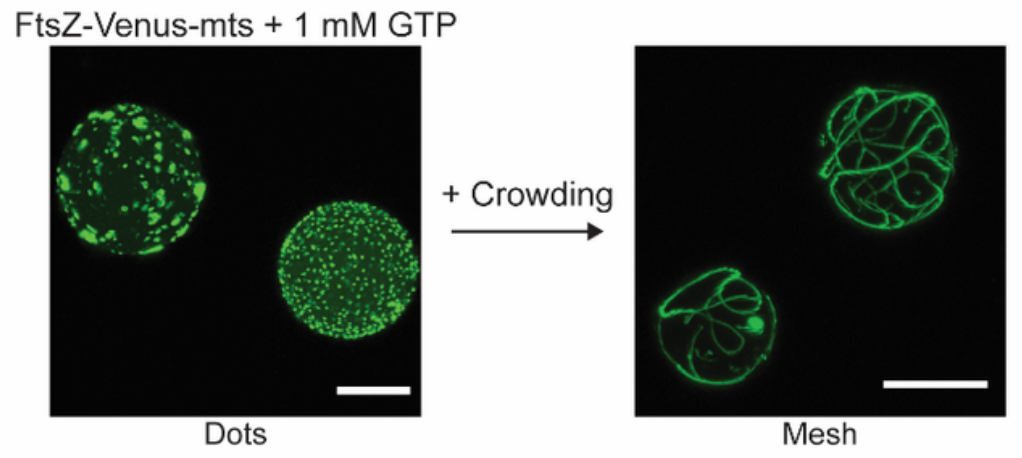

C

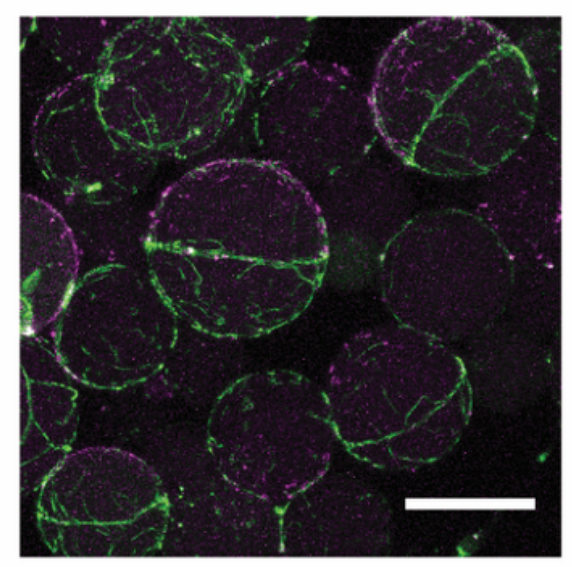

d

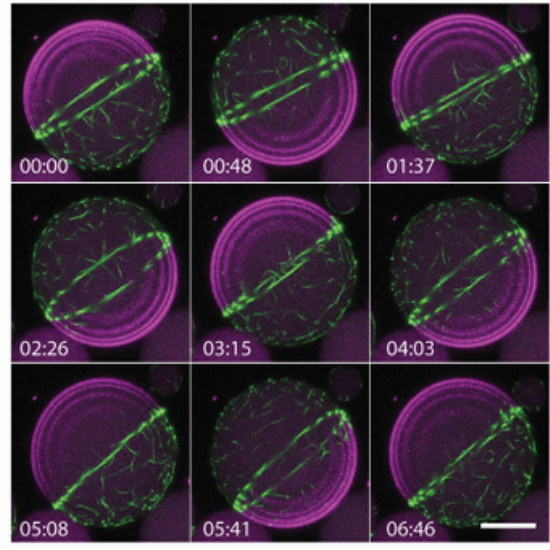

$30 \mathrm{~min}$

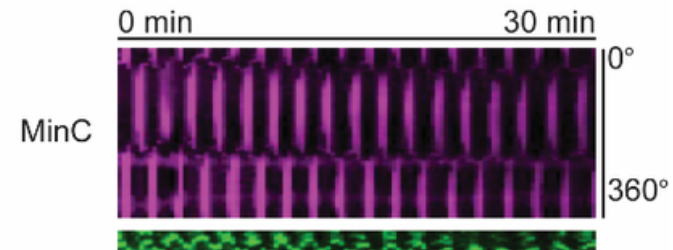

FtsZ

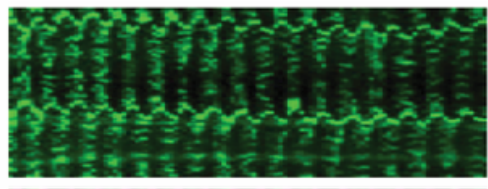

Merged

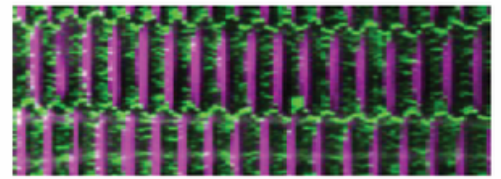

e

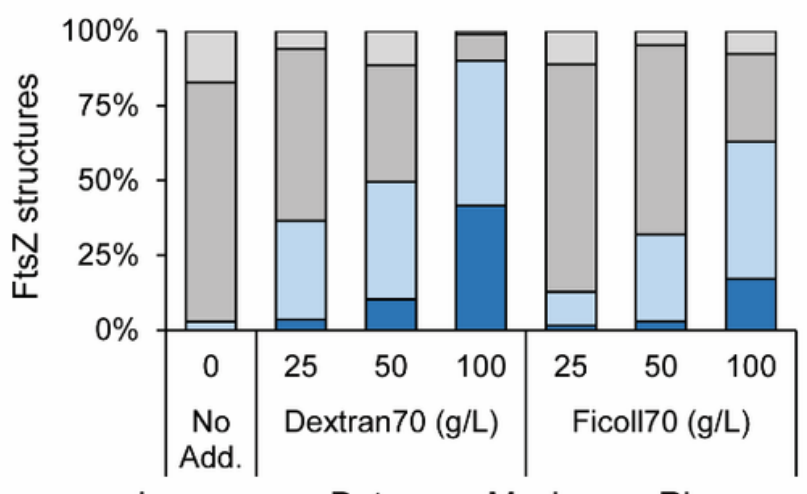

口Lumen aDots aMesh aRings f

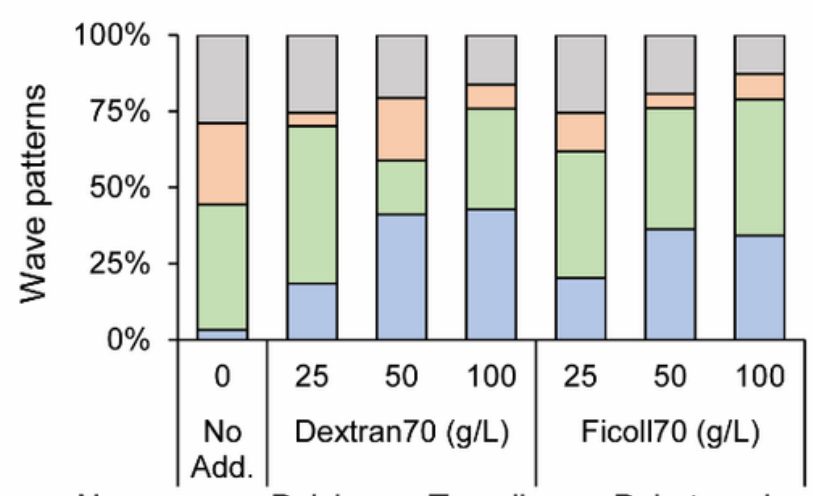

$\square$ Nowaves $\square$ Pulsing $\square$ Traveling $\square$ Pole-to-pole

Figure 2 
Reconstitution of FtsZ-ring structures inside lipid vesicles. A Schematic illustration of the FtsZ-Venus-mts system. B 3D max projection of encapsulated FtsZ-Venus-mts (green) in the absence (left) or presence (right) of macromolecular crowding using $100 \mathrm{~g} / \mathrm{L}$ Dextran70 and $1 \mathrm{mM}$ GTP. Scale bar $15 \mu \mathrm{m}$. c 3D max projection of a merged confocal image of vesicles containing the MinCDE system and FtsZ-Venus-mts in the presence of $100 \mathrm{~g} / \mathrm{L}$ Dextran70. The Min-assisted formation of pronounced FtsZ-rings out of isotropic meshwork can be clearly observed. Magenta and green correspond to mScarlet-I-MinC and FtsZ-Venus$\mathrm{mts}$, respectively. Scale bar $25 \mu \mathrm{m}$. d Time-lapse images of the FtsZ-ring structure stabilized by pole-topole oscillations of Min waves. 3D max projection of merged images of mScarlet-I-MinC and FtsZ-Venus$\mathrm{mts}$. Scale bar $15 \mu \mathrm{m}$. Time represented in min:sec. Right: Kymographs of the FtsZ-ring placement by a pole-to-pole oscillation wave captured by mScarlet-I-MinC (magenta) and FtsZ-Venus-mts (green). E Frequency of FtsZ-ring formation in co-reconstituted vesicles. FtsZ structures were differentiated into four categories: rings, mesh, dots, or luminal localization $(n=180,252,615,473,250,366,543$ for no additives, 25, 50, $100 \mathrm{~g} / \mathrm{L}$ Dextran70, 25, 50, $100 \mathrm{~g} / \mathrm{L}$ Ficoll70, respectively). F Frequency of emerging Min wave patterns (pole-to-pole oscillations, traveling waves, pulsing, or absence of waves) inside vesicles containing the MinCDE system co-reconstituted with FtsZ-Venus-mts among the addition of different macromolecular crowding concentrations using Dextran70 or Ficoll70 ( $n=90,157,172,289,227,391$, 289 for no additives, 25, 50, $100 \mathrm{~g} / \mathrm{L}$ Dextran70, 25, 50, $100 \mathrm{~g} / \mathrm{L}$ Ficoll70, respectively). The occurrence of pole-to-pole oscillations rises in parallel to the formation of equatorial FtsZ-rings, indicating a positive feedback between the two phenomena. 
a
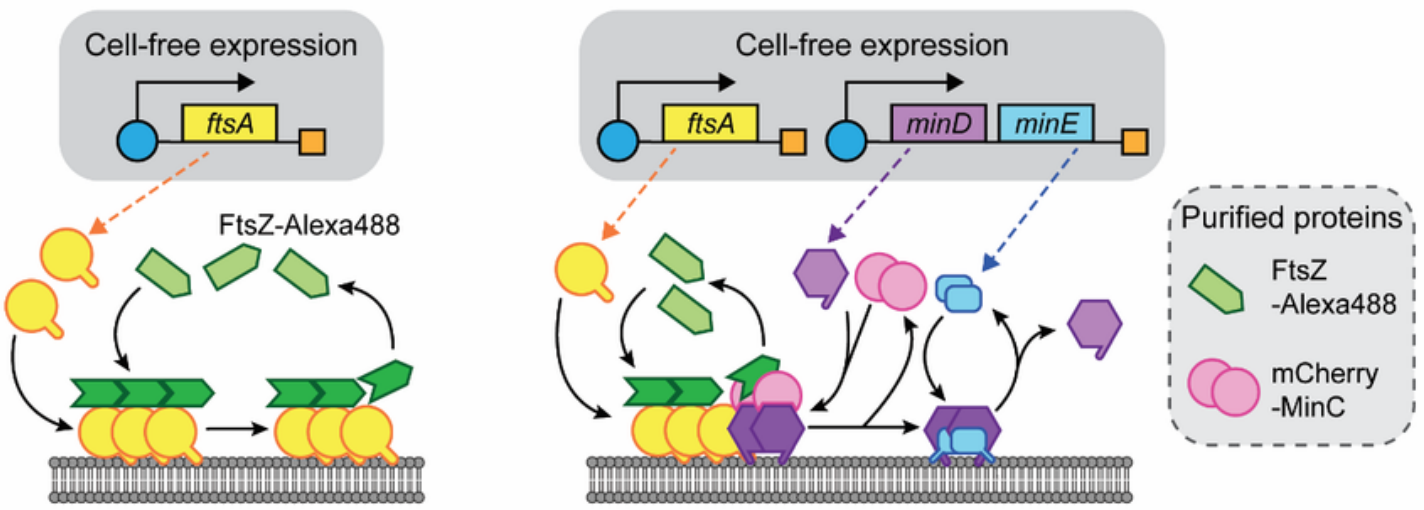

b
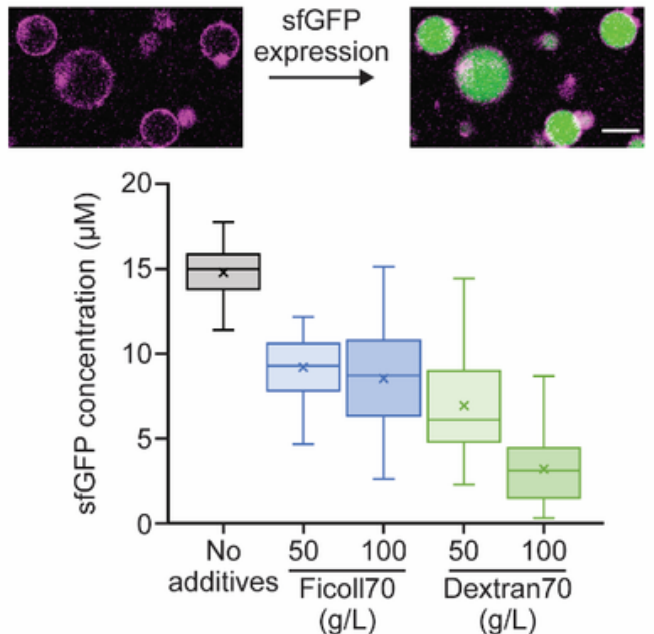

d

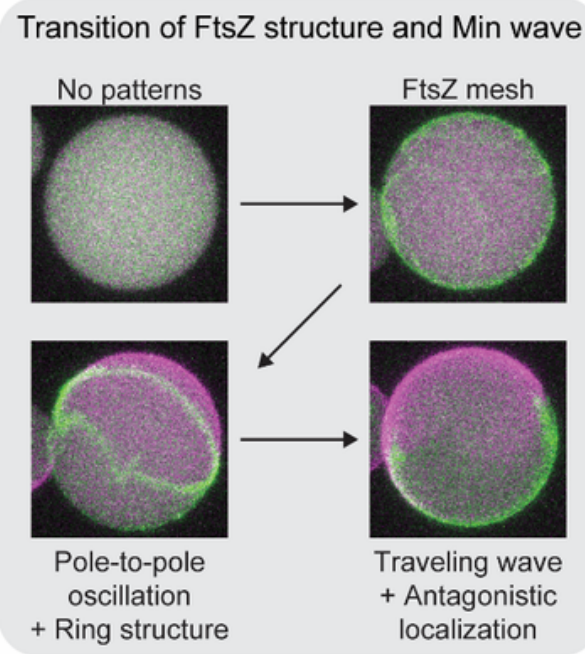

C
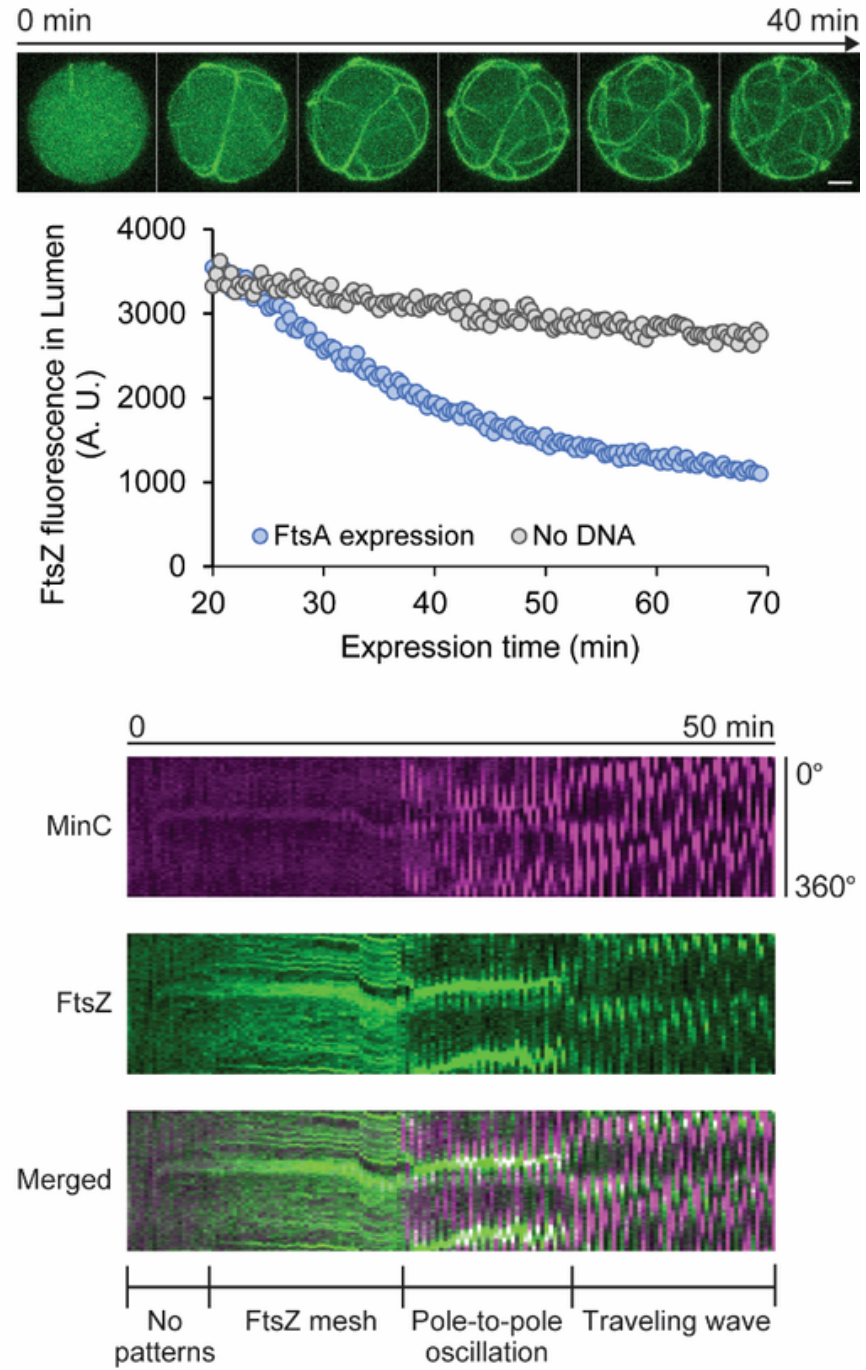

\section{Figure 3}

Reconstitution of FtsZ-FtsA mesh and Min wave regulated FtsZ-ring structures via cell-free expression. a Schematic illustrations of PURE cell-free expression system. Left: Expression of FtsA with purified FtsZ. Right: Co-expression of FtsA, MinD, and MinE with purified FtsZ-Alexa488 and mCherry-MinC. b Cell-free expression of sfGFP within vesicles under different crowding conditions. Top: Expression of sfGFP under $50 \mathrm{~g} / \mathrm{L}$ of Ficoll7. Images show the same vesicles before incubation and after 200 min incubation at 37 
${ }^{\circ} \mathrm{C}$. Scale bar: $20 \mu \mathrm{m}$. Bottom: Box plot of expression levels of sfGFP inside vesicles after $200 \mathrm{~min}$ incubation at $37^{\circ} \mathrm{C}$. Under Ficoll70 crowding, sfGFP expression level reached around $10 \mu \mathrm{M}$, while Dextran70 radically impaired expression. Mean is shown as a cross mark, box limits are quartiles 1 and 3 , line inside boxes indicate median, and whiskers are the highest or lowest data points. $n=100$ for each crowding condition. c FtsZ-FtsA mesh structure inside vesicles via FtsA expression. Top: Time-lapse images of FtsZ mesh structure formation with 3D max projection. FtsZ indicates dynamic assembly into bundles on the membrane inside the vesicle along with FtsA expression. Bottom: localization change of FtsZ-Alexa488 along with FtsA expression in the lumen of the vesicles. FtsZ level decreases in the lumen by expressing FtsA, targeting FtsZ to the membrane, while the negative control (no DNA) indicates a slight decrease of fluorescence by photobleaching. Scale bar: $5 \mu \mathrm{m}$. d Regulation of FtsZ localization by Min waves inside vesicles. Left: 3D max projection of the transition of FtsZ structures and Min wave patterns by co-expression of FtsA, MinD, and MinE within vesicles. FtsZ and Min proteins transit from no patterns to an FtsZ mesh on the membrane, FtsZ-ring structure regulated by pole-to-pole oscillations of Min waves, and antagonistic localization of Min traveling waves and FtsZ mesh. Right: Kymographs of the pattern transition of Min waves and FtsZ structures. mCherry-MinC and FtsZ-Alexa488 are indicated in magenta and green, respectively. 

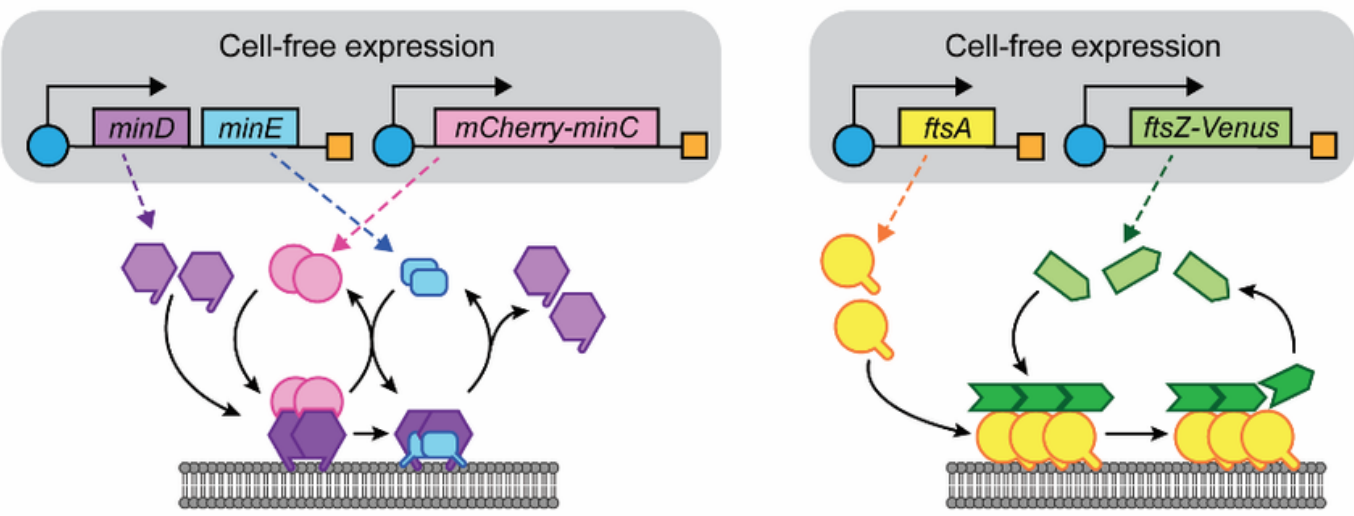

b
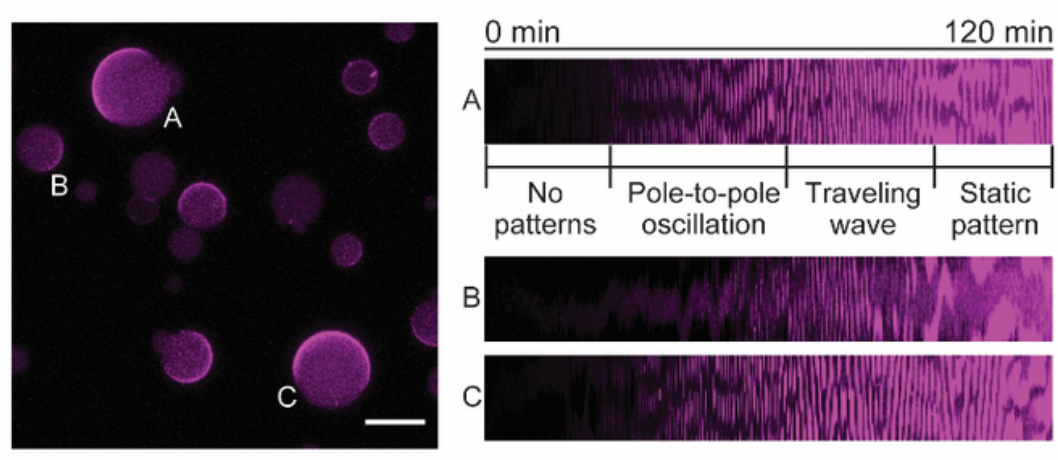

d

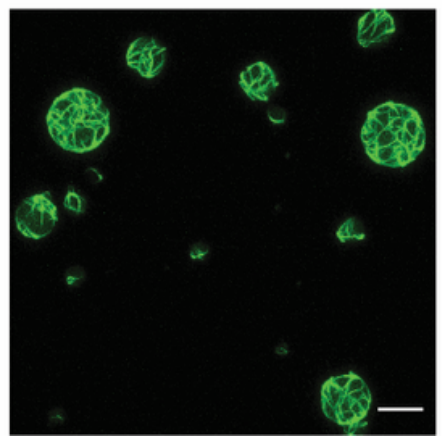

e

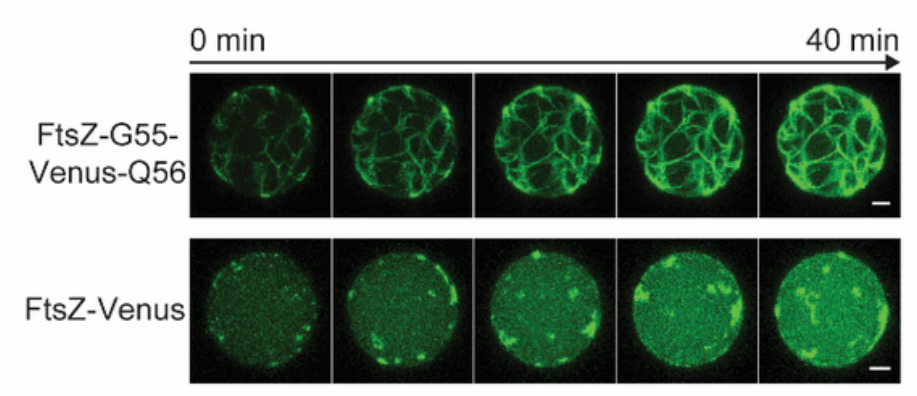

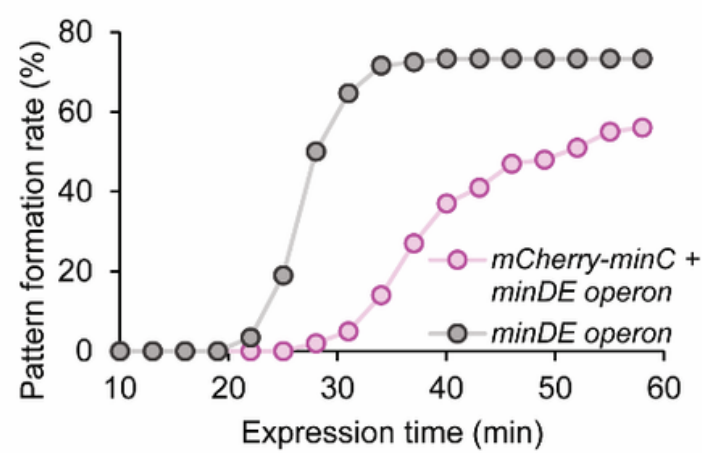

f

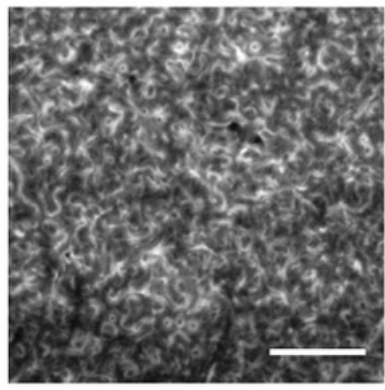

\section{Figure 4}

Reconstitution of sub-division systems by co-expressing all essential components. a Schematic illustrations of co-expression systems. Left: Co-expression of mCherry-MinC, MinD, and MinE. Right: Coexpression of FtsA and FtsZ-Venus mutant. $\mathbf{b}$ Emergence of Min waves by cell-free expressed mCherryMinC, MinD, and MinE inside vesicles. Left: 3D max projection of Min wave pattern formation inside vesicles after $2 \mathrm{~h}$ expression of $\mathrm{mCherry}-\mathrm{MinC}, \mathrm{MinD}$, and MinE at $37^{\circ} \mathrm{C}$. Right: Kymographs of the Min waves inside vesicles correspond to the same letters in the left image captured by mCherry-MinC fluorescence. Wave dynamics were highly sensitive to MinDE concentrations. Scale bar: $20 \mu \mathrm{m}$. c Timedependent occurrence of Min wave patterns inside lipid vesicles. Percentage of Min patterns (dynamic waves and static patterns) among vesicles are calculated every 3 min after 10 min incubation. minDE operon (black points and gray lines) for MinDE co-expression, mCherry-MinC + minDE operon (magenta points and pink lines) for MinCDE co-expression. $n=116$ and 100 for MinDE and MinCDE expression, respectively. $\mathbf{d}$ 3D max projection of the FtsZ mesh structures inside vesicles after $1 \mathrm{~h}$ co-expression of 
FtsZ-G55-Venus-Q56 and FtsA at $37^{\circ} \mathrm{C}$. Scale bar: $20 \mu \mathrm{m}$. e Comparison of FtsZ mesh formation dynamics for FtsZ-G55-Venus-Q56 and FtsZ-Venus. FtsZ-G55-Venus-Q56 efficiently forms mesh structures on the membrane via co-expression with FtsA, while FtsZ-Venus forms dot-like structures. Scale bars: $5 \mu \mathrm{m}$. f FtsZ-G55-Venus-Q56 structures on SLBs. FtsZ-G55-Venus-Q56 was anchored to the membrane through FtsA and assembled into dynamic vortices similar to wild-type FtsZ (Supplementary Fig. 5d). Scale bar: $10 \mu \mathrm{m}$.

a

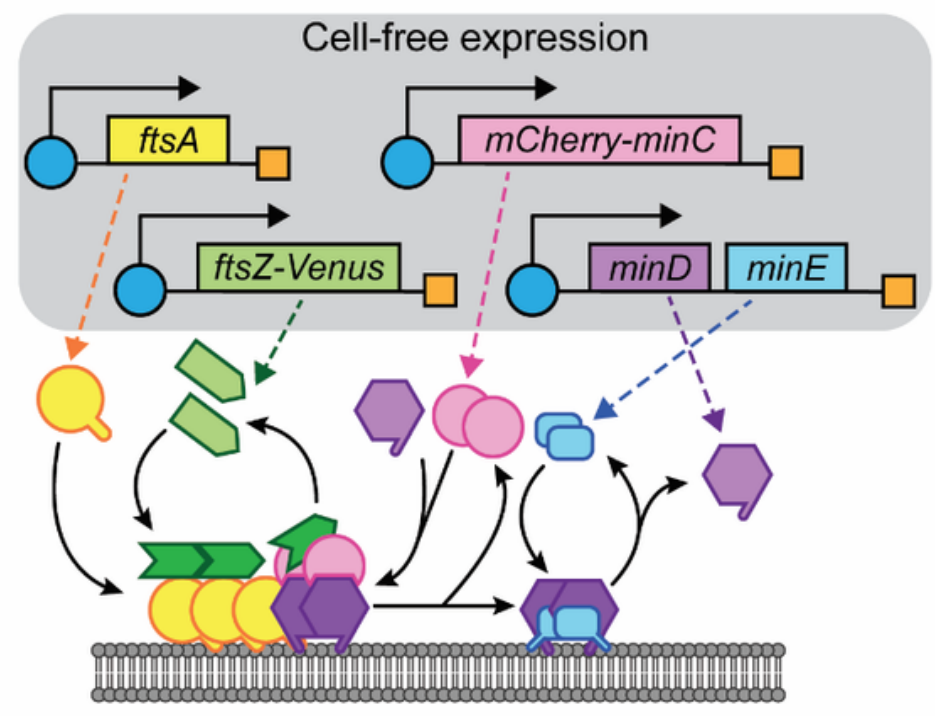

b

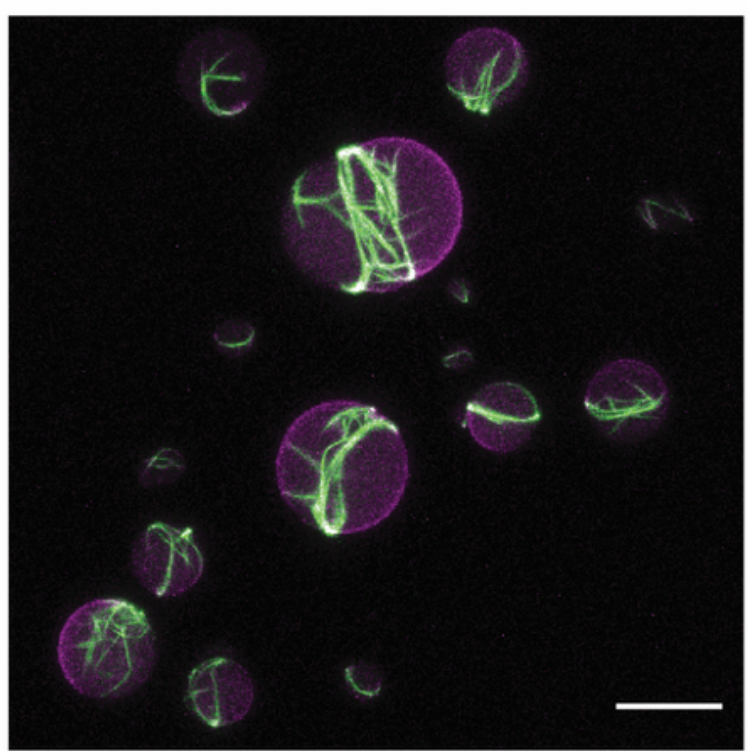

C $0 \mathrm{~min}$ 75 min

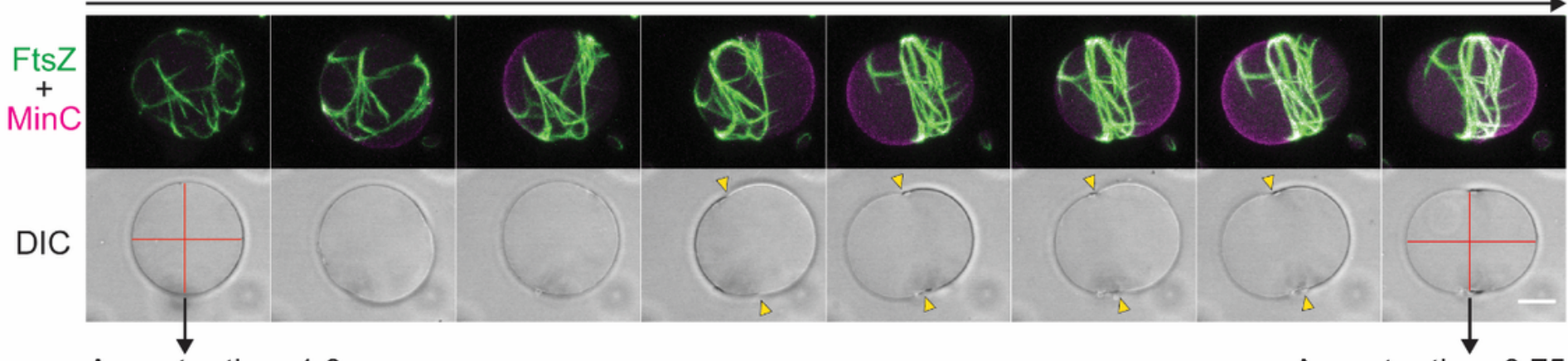

Aspect ratio $\approx 1.0$

Aspect ratio $\approx 0.75$

d

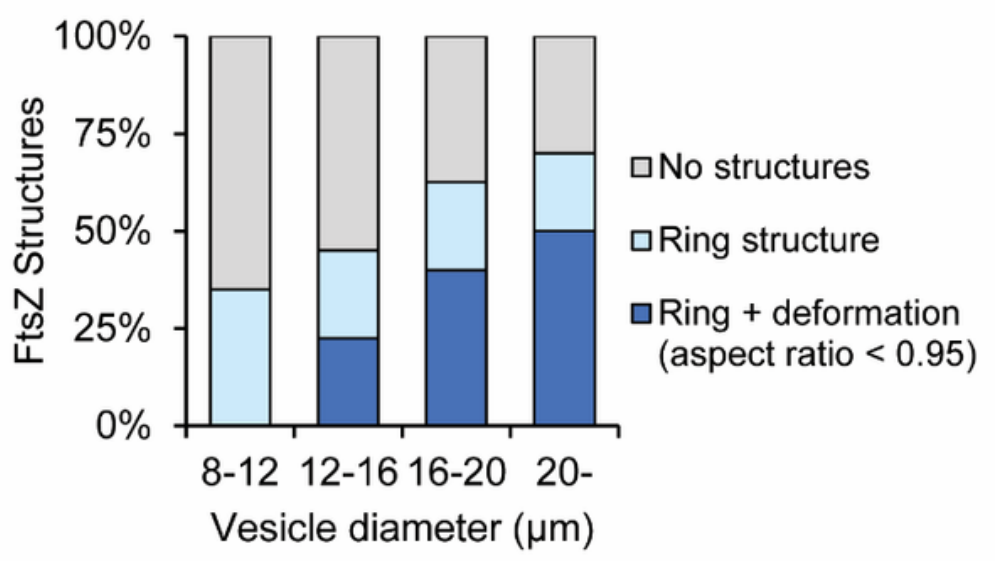

e

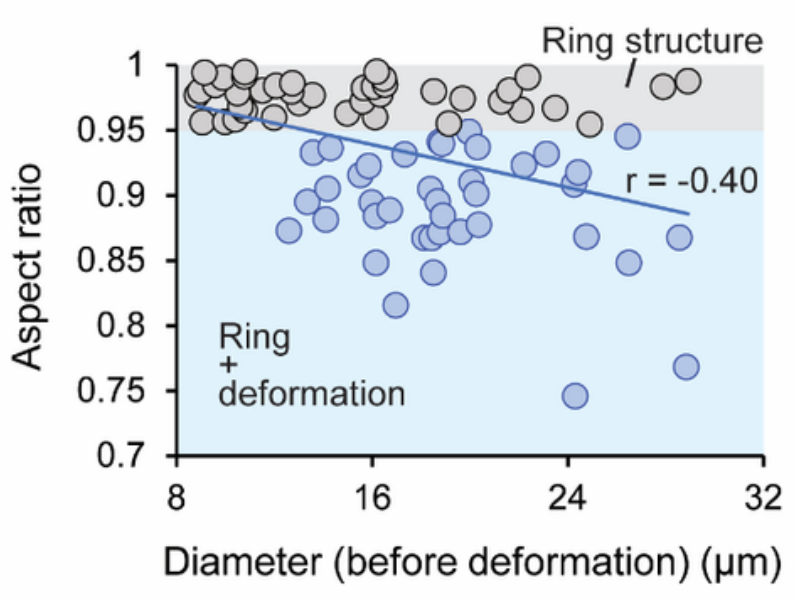




\section{Figure 5}

Reconstitution of minimal division ring placement system by co-expression of five essential components. A Schematic illustration of co-expression systems of FtsA, FtsZ-G55-Venus-Q56, mCherry-MinC, MinD, and MinE. B 3D max projection of FtsZ-ring formation and Min protein localization at the pole of the vesicles after 100 min expression at $37^{\circ} \mathrm{C}$. mCherry-MinC and FtsZ-G55-Venus-Q56 are indicated in magenta and green, respectively. Scale bar: $20 \mu \mathrm{m}$. c Time-lapse images of the formation of the FtsZ-ring structure and constriction of the vesicle driven by the pole-to-pole oscillations of MinCDE. Top panels: 3D max projection of the merged image of mCherry-MinC (magenta) and FtsZ-G55-Venus-Q56 (green). The isotropic FtsZ mesh is condensed into a ring structure after the emergence of pole-to-pole oscillations, which later constricts the vesicle membrane. Bottom panels: Differential interference contrast (DIC) images of the equatorial plane of the vesicle. Red lines indicate aspect ratios of the vesicle before and after constriction by FtsZ-ring, yellow triangles indicate necks of the constriction by FtsZ-ring. Scale bar: $10 \mu \mathrm{m}$. $\mathbf{d}$ Percentage of the ring structure and deformed vesicles among different vesicle sizes after 2-3 $\mathrm{h}$ co-expression of 5 essential genes at $37^{\circ} \mathrm{C}$. Proportions of both FtsZ-ring structures and deformation of the vesicles increase along vesicle diameter, while deformation never appears under $12 \mu \mathrm{m}$ in a diameter. $\mathrm{N}=40$ for each diameter range. $\mathrm{E}$ Scatter plot of the aspect ratios of deformed vesicles against vesicle diameter after 2-3 h co-expression of 5 essential genes at $37^{\circ} \mathrm{C}$. The correlation coefficient shows moderate correlation (Pearson's $r=-0.40$ ), suggesting greater effective force generation by FtsZ-rings within larger vesicles. Gray and blue dots (with respective background colors) indicate non-deformed vesicles (aspect ratio $>0.95$ ) and deformed vesicles (aspect ratio $<0.95$ ), respectively. $N=79$.

\section{Supplementary Files}

This is a list of supplementary files associated with this preprint. Click to download.

- SupplementaryText.docx

- Supplementarymovie1.avi

- Supplementarymovie2.avi

- Supplementarymovie3.avi

- Supplementarymovie4.avi

- Supplementarymovie5.avi

- Supplementarymovie6.avi

- Supplementarymovie7.avi

- Supplementarymovie8.avi 